TRANSACTIONS OF THE

AMERICAN MATHEMATICAL SOCIETY

Volume 364, Number 2, February 2012, Pages 571-594

S 0002-9947(2011)05161-5

Article electronically published on September 14,2011

\title{
TANGENT ALGEBRAS
}

\author{
ARON SIMIS, BERND ULRICH, AND WOLMER V. VASCONCELOS
}

\begin{abstract}
One studies the Zariski tangent cone $T_{X} \stackrel{\pi}{\longrightarrow} X$ to an affine variety $X$ and the closure $\bar{T}_{X}$ of $\pi^{-1}(\operatorname{Reg}(X))$ in $T_{X}$. One focuses on the comparison between $T_{X}$ and $\bar{T}_{X}$, giving sufficient conditions on $X$ in order that $T_{X}=\bar{T}_{X}$. One considers, in particular, the question of when this equality takes place in the presence of the reducedness of the Zariski tangent cone. Another problem considered here is to understand the impact of the Cohen-Macaulayness or normality of $\bar{T}_{X}$ on the local structure of $X$.
\end{abstract}

\section{INTRODUCTION}

Let $A$ be an affine algebra over a field $k$, and let $\Omega_{A / k}$ be the $A$-module of Kähler $k$-differentials. Classically, the properties of this module are closely related to the local singularities of $A$, embodying in particular the well-known Jacobian criterion for the smoothness of $A$. Its sheaf version for an algebraic variety is fundamental in intersection theory, and its cohomology is a major vehicle for the study of the global geometry of the variety.

In this paper we focus on two basic algebras associated to $\Omega_{A / k}$, where $A$ is a reduced ring essentially of finite type over a perfect field $k$ : the Rees algebra $\mathbb{R}_{A / k}$ of $\Omega_{A / k}$ (defined in Section 2) and its close predecessor $\mathbb{S}_{A / k}$, the symmetric algebra of $\Omega_{A / k}$. If $A$ is regular these algebras coincide, but otherwise they may be quite apart and their respective properties have different impact on the nature of $A$. In 22 the ring $\mathbb{S}_{A / k}$ was called the Zariski tangent algebra of $A$ because the closed fibers of the map $\operatorname{Spec}\left(\mathbb{S}_{A / k}\right) \longrightarrow \operatorname{Spec}(A)$ are the Zariski tangent spaces to closed points of $\operatorname{Spec}(A)$, when $A$ is an affine algebra over an algebraically closed field. Alternatively, $\operatorname{Spec}\left(\mathbb{S}_{A / k}\right)$ is the first jet scheme of $\operatorname{Spec}(A)$. As to $\mathbb{R}_{A / k}$, it plays the role of the coordinate ring of a correspondence in biprojective space (see [19]). A variation on $\mathbb{R}_{A / k}$ is the Rees algebra of the top wedge product of $\Omega_{A / k}$ which, as was argued in [21, gives a full-fledged algebraic version of Zak's inequality for the dimension of the Gauss image of a projectively embedded variety.

Yet another version of such "tangent" algebras is the tangent star algebra $\mathbb{T}_{A / k}$, defined as the associated graded ring of the kernel $\mathbb{D}_{A / k}$ of the multiplication map $A \otimes_{k} A \longrightarrow A$, the latter also known as the diagonal ideal. Looking at the relation

Received by the editors November 25, 2007 and, in revised form, June 18, 2009.

2010 Mathematics Subject Classification. Primary 13A30, 13N05; Secondary 13B22, 14F10.

The first author was partially supported by CNPq, Brazil.

The second author was partially supported by the NSF, USA.

The third author was partially supported by the NSF, USA.

(C)2011 American Mathematical Society 
of this algebra to $\mathbb{S}_{A / k}$ was the core of [22]. Under mild conditions, it can be shown that $\mathbb{T}_{A / k}$ modulo its $A$-torsion is isomorphic to $\mathbb{R}_{A / k}$ (see [19, 4.6]). Therefore a certain knowledge about the structure of $\mathbb{R}_{A / k}$ might be borrowed from results in 22. However, since this would not make up for the main bulk in the present work, we have decided to study $\mathbb{R}_{A / k}$ ab initio, in its own right.

There is a natural surjection

$$
\mathbb{S}_{A / k} \rightarrow \mathbb{R}_{A / k} .
$$

A great deal of the present work has to do with this map, whose kernel measures the failure of describing $\mathbb{R}_{A / k}$ solely in terms of linear equations, or to use a recent terminology for modules, the failure of $\Omega_{A / k}$ being of linear type. We also study how the singularities of $A$ are reflected in the normality, Cohen-Macaulayness and Gorensteiness of $\mathbb{R}_{A / k}$.

Let us now describe the main results in some detail.

The paper is divided into three sections. In the first section we focus on the Zariski tangent algebra $\mathbb{S}_{A / k}$. Recall that $\Omega_{A / k} \simeq \mathbb{D}_{A / k} / \mathbb{D}_{A / k}^{2}$. Now, quite generally, let $S$ be a Noetherian ring and let $\mathbf{D} \subset S$ be an ideal such that the symmetric algebra $\mathcal{S}_{A}\left(\mathbf{D} / \mathbf{D}^{2}\right)$ is torsionfree over $A=S / \mathbf{D}$. If one assumes, moreover, that $\mathbf{D}$ is generically a complete intersection and $A$ is reduced, then $\mathcal{S}_{A}\left(\mathbf{D} / \mathbf{D}^{2}\right)$ is reduced. The converse to this statement, namely, that $\mathcal{S}_{A}\left(\mathbf{D} / \mathbf{D}^{2}\right)$ is torsionfree if it is reduced, is known to be false in general. The corresponding question for the associated graded ring $\operatorname{gr}_{\mathbf{D}}(S)$ was treated in [14,1.10] and shown to be affirmative provided $S$ is a quasi-unmixed local ring and $A$ has finite projective dimension over $S$. Although the diagonal ideal $\mathbb{D}_{A / k}$ does not have finite projective dimension for singular $A$, our goal in the first part of the section is to consider this converse in the framework of $\mathbb{S}_{A / k}=\mathcal{S}_{A}\left(\mathbb{D}_{A / k} / \mathbb{D}_{A / k}^{2}\right)$, trading off the homological restriction on $A$ for a condition on its defining equations (Theorem 2.1 and Corollary 2.2).

In the second part of this section we deal with the same question in the case of an analytically irreducible curve singularity over an algebraically closed field. Here, without any assumptions on the defining equations, we prove that such curves are non-singular provided $\mathbb{S}_{A / k}$ is reduced (Theorems 2.6] and 2.8). This can be regarded as an analogue of Berger's conjecture (see [3]) in which the reducedness of $\mathbb{S}_{A / k}$ replaces the torsionfreeness of $\Omega_{A / k}$.

In the last part of the section we study the relationship between the normality of $\mathbb{S}_{A / k}$ and its "reflexivity", defined in terms of the double duals of the symmetric powers of $\Omega_{A / k}$. Quite generally, the latter is known to imply the former, at least when $A$ is normal. We will prove that the converse holds under suitable conditions on the equations of order 2 defining $A$ (Theorem 2.10).

The second section is mainly devoted to the behavior of the Rees algebra $\mathbb{R}_{A / k}$. Our first fundamental result deals with the case where $A$ is locally everywhere a complete intersection. Notice that, in this case, the module of differentials $\Omega_{A / k}$ has projective dimension at most one. We first recall in parallel the case of the symmetric algebra. Namely, if $A$ is a local Cohen-Macaulay ring and $E$ is a finite $A$-module of projective dimension at most one, then $E$ satisfies condition $\left(F_{0}\right)$ if and only if the symmetric algebra $\mathcal{S}_{A}(E)$ is a complete intersection over $A$, and $E$ satisfies condition $\left(F_{1}\right)$ if and only if $\mathcal{S}_{A}(E)$ is $A$-torsionfree ([1, Proposition 4], [13, 1.1], 24, 3.4]); see Section 2 for an explanation of the condition $\left(F_{t}\right)$. As the torsionfreeness of $\mathcal{S}_{A}(E)$ gives a natural isomorphism $\mathcal{S}_{A}(E) \simeq \mathcal{R}_{A}(E)$, it follows that 
condition $\left(F_{1}\right)$ implies the Rees algebra $\mathcal{R}_{A}(E)$ to be Cohen-Macaulay. However, the converse does not hold in general, even if one assumes the preliminary condition $\left(F_{0}\right)$. Nevertheless we are able to prove this converse in the case where $E$ is the module of differentials of a complete intersection over a field of characteristic zero (Theorem 3.1). Our result shows that Cohen-Macaulayness is a rather restrictive property for $\mathbb{R}_{A / k}$. It implies for instance that if $X \subset \mathbb{P}_{k}^{2 d+1}$ is a $d$-dimensional non-degenerate smooth complete intersection over a field $k$ of characteristic zero with homogeneous coordinate ring $A$, then $\mathbb{R}_{A / k}$ is never a Cohen-Macaulay ring. It would be interesting to have a geometric explanation for this phenomenon.

The second kind of structural results concerns the normality of $\mathbb{R}_{A / k}$, in which the condition $\left(F_{2}\right)$ will play a predominant role. Throughout this part $A$ is assumed to be a normal domain. To enlarge the picture we consider yet another algebra associated to a finite $A$-module $E$, namely, $\mathcal{B}_{A}(E):=\bigoplus_{i \geq 0}\left(E^{i}\right)^{* *}$, where $E^{i}$ denotes the $i$ th graded component of $\mathcal{R}_{A}(E)$ and $-{ }^{*}$ stands for $\operatorname{Hom}(-, A)$. This algebra could be dubbed the reflexive closure of the Rees algebra $\mathcal{R}_{A}(E)$ - note it is a Krull domain with the same divisor class group as $A$, but may fail to be Noetherian (see, e.g., [17, Theorem 2]). This algebra has been studied earlier in [11] (see also [28, Chapter 7]). First is a basic criterion for the equality $\mathcal{R}_{A}(E)=\mathcal{B}_{A}(E)$, assuming that $\mathcal{R}_{A}(E)$ is normal or just satisfies Serre's condition $\left(S_{2}\right)$ (Proposition 3.4). The criterion is given in terms of a new condition that controls the growth of the local analytic spreads of $E$. We then move on to an encore of modules of projective dimension at most one over normal Cohen-Macaulay domains. For such a module $E$ it is known that condition $\left(F_{2}\right)$ holds if and only if $\mathcal{S}_{A}(E)=\mathcal{B}_{A}(E)$ (see [1, Proposition 4]). In particular, if $E$ satisfies $\left(F_{2}\right)$, then $\mathcal{R}_{A}(E)$ is normal. Surprisingly, the converse holds as well, provided the non-free locus of $E$ be contained in the singular locus of $A$ (Theorem 3.7). An application to $\Omega_{A / k}$ when $A$ is a normal complete intersection now ensues (Corollary 3.8). In fact it turns out that the inequalities edim $A_{\mathfrak{p}} \leq 2 \operatorname{dim} A_{\mathfrak{p}}-2$ locally on the singular locus of $A$ become equivalent to the powers of $\Omega_{A / k}$ being integrally closed in the range $1 \leq i \leq \operatorname{ecodim} A$; here $\operatorname{ecodim} A:=\operatorname{edim} A-\operatorname{dim} A$.

In the last section we consider special situations. A substantial part thereof deals with extending the previous results to cases other than complete intersections. A reasonable bulk of results is obtained if $A$ is of low dimension or low embedding codimension or is sufficiently structured. Thus we find satisfactory answers in the codimension 2 perfect and codimension 3 Gorenstein cases (Proposition 4.1, Theorem 4.8 and Proposition 4.2. . Naturally this part requires several developments, and the results become somewhat too technical to be described in this introduction. We refer the reader to the appropriate places in the last part of this work.

The section ends with some consequences of the theory so far regarding its relation to Calabi-Yau varieties. We argue that if $A$ is the homogeneous coordinate ring of a Calabi-Yau variety, then often $\mathbb{S}_{A / k}$ or $\mathbb{R}_{A / k}$ are the homogeneous coordinate rings of "Calabi-Yau like" varieties (Proposition 4.9 and Proposition 4.10).

\section{The Symmetric Algebra of the Module of DifFerentials}

We need some preliminaries on symmetric and Rees algebras of modules. We will draw upon 23] for terminology and basic notions about Rees algebras of modules. Let $E$ be a finite module over a Noetherian ring $A$ and assume that $E$ is generically 
free (i.e., free locally at every associated prime of $A$ ). In this setting the Rees algebra $\mathcal{R}_{A}(E)$ of $E$ is defined as the symmetric algebra of $E$ modulo $A$-torsion, $\mathcal{R}_{A}(E):=\mathcal{S}_{A}(E) / \tau_{A}\left(\mathcal{S}_{A}(E)\right)$. As is known, this definition retrieves the usual notion of the Rees algebra of an ideal containing a regular element. If in addition $E$ is torsionfree, then $E$ can be embedded into a finite free module $F$ and the Rees algebra of $E$ can be identified with the image of $\mathcal{S}_{A}(E)$ in the polynomial ring $\mathcal{S}_{A}(F)$. In case $\mathcal{S}_{A}(E)=\mathcal{R}_{A}(E)$, we say that $E$ is a module of linear type.

We recall the following additional notions. Let $E$ be a finite module over a Noetherian ring $A$. Suppose that $E$ has a rank $r$ (i.e., $E$ is free of rank $r$ locally at every associated prime of $A$ ). Given an integer $t \geq 0$, we say that $E$ satisfies condition $\left(F_{t}\right)$ if $\mu\left(E_{\mathfrak{p}}\right) \leq \operatorname{dim} A_{\mathfrak{p}}+r-t$ for every $\mathfrak{p} \in \operatorname{Spec}(A)$ such that $E_{\mathfrak{p}}$ is not free; here $\mu(-)$ denotes the minimal number of generators. In terms of Fitting ideals this condition means that height $\operatorname{Fitt}_{i}(E) \geq i-r+t+1$ for $i \geq r$. The properties $\left(F_{t}\right)$ play a role in the study of $\mathcal{S}_{A}(E)$. Thus, e.g., the Cohen-Macaulayness of this algebra implies condition $\left(F_{0}\right)$ for $E$, and if $E$ is of linear type, then $\left(F_{1}\right)$ holds.

In a similar vein one can introduce yet another condition based on the analytic spread $\ell(E)$ of a finite module $E$ over a local ring $(A, \mathfrak{m})$, the latter defined to be the Krull dimension of the residue algebra $\mathcal{R}_{A}(E) / \mathfrak{m} \mathcal{R}_{A}(E)$ (see [23]). We will say that a finite module $E$ of rank $r$ over a Noetherian ring $A$ satisfies condition $\left(L_{t}\right)$ if $\ell\left(E_{\mathfrak{p}}\right) \leq \operatorname{dim} A_{\mathfrak{p}}+r-t$ for every $\mathfrak{p} \in \operatorname{Spec}(A)$ with $\operatorname{dim} A_{\mathfrak{p}} \geq t$. Roughly, this condition plays a similar role for the Rees algebra as $\left(F_{t}\right)$ plays for the symmetric algebra. Condition $\left(F_{t}\right)$ implies $\left(L_{t}\right)$, and the converse holds if $E$ is free locally in codimension $\leq t-1$ and is of linear type. We will only use these conditions in the range $0 \leq t \leq 2$.

Our main interest throughout is the module of Kähler differentials $\Omega_{A / k}$. Let $A$ be a reduced algebra essentially of finite type over a perfect field $k$; in particular, $\Omega_{A / k}$ is generically free. We denote by $\mathbb{S}_{A / k}:=\mathcal{S}_{A}\left(\Omega_{A / k}\right)$ and by $\mathbb{R}_{A / k}:=$ $\mathcal{R}_{A}\left(\Omega_{A / k}\right)$ the symmetric algebra and the Rees algebra of $\Omega_{A / k}$, respectively. If in addition $(A, \mathfrak{m})$ is local and equidimensional, then $\Omega_{A / k}$ has a rank, given by

$$
\operatorname{rank} \Omega_{A / k}=\operatorname{dim} A+\operatorname{trdeg}_{k}(A / \mathfrak{m}) .
$$

In this case the above Fitting conditions can be expressed in terms of local embedding dimensions. Namely, $\Omega_{A / k}$ satisfies $\left(F_{t}\right)$ if and only if edim $A_{\mathfrak{p}} \leq 2 \operatorname{dim} A_{\mathfrak{p}}-t$ for every non-regular prime $\mathfrak{p} \in \operatorname{Spec}(A)$ (see [22, the proof of 2.3]). The same characterization holds when $A$ is a not necessarily local domain.

In the following part we put an emphasis on the structure of the symmetric algebra $\mathbb{S}_{A / k}$ as an ancestor of the corresponding Rees algebra $\mathbb{R}_{A / k}$, which will be the topic of the next section.

2.1. Reducedness: Relation to torsionfreeness. The main result of this part has a curious geometric consequence. Let $A$ be a local domain with an isolated singularity that is essentially of finite type over a perfect field. If the Zariski tangent algebra of $A$ is reduced, but not a domain, then the defining ideal of $A$ has to contain "many" quadrics. In the language of jet schemes, the same conclusion holds if the first jet scheme of $\operatorname{Spec}(A)$ is reduced, but not irreducible.

Theorem 2.1. Let $A$ be a reduced ring essentially of finite type over a perfect field $k$. Assume that for every non-minimal $\mathfrak{p} \in \operatorname{Spec}(A)$ there is a presentation $A_{\mathfrak{p}} \simeq R / I$, where $(R, \mathfrak{n})$ is a regular local ring essentially of finite type over $k$ and 
$I \subset R$ is an ideal satisfying

$$
\mu\left(I+\mathfrak{n}^{3} / \mathfrak{n}^{3}\right) \leq \operatorname{dim} R-1 .
$$

Then $\mathbb{S}_{A / k}$ is reduced (if and) only if it is A-torsionfree (i.e., $\Omega_{A / k}$ is of linear type).

Proof. The "if" statement was explained in the Introduction.

To show the converse, let $\mathcal{T} \subset \mathbb{S}_{A / k}$ denote the $A$-torsion submodule of $\mathbb{S}_{A / k}$ and suppose that $\mathcal{T} \neq 0$. Then there exists an associated, hence minimal, prime of $\mathbb{S}_{A / k}$ contracting to a non-minimal prime of $A$. We may further take such a non-minimal prime to be minimal among all non-minimal primes of $A$ that are contracted from some minimal prime of $\mathbb{S}_{A / k}$. By localizing at this non-minimal prime, we do not change either the hypotheses or the conclusion of the statement, so we can reduce the argument to the situation where $(A, \mathfrak{m}, K)$ is local and $\mathfrak{m}$ is the contraction of a minimal prime of $\mathbb{S}_{A / k}$. Moreover, every minimal prime of $\mathbb{S}_{A / k}$ not containing $\mathfrak{m}$ must contract to a minimal prime of $A$, and hence contains the torsion $\mathcal{T}$. From this follows, since $\mathbb{S}_{A / k}$ is reduced, the crucial relation

$$
\mathcal{T} \cap \mathfrak{m} \mathbb{S}_{A / k}=0 .
$$

Now $\mathbb{S}_{A / k} / \mathfrak{m} \mathbb{S}_{A / k}$ is a standard graded polynomial ring over $K$ in $n:=\mu\left(\Omega_{A / k}\right)$ variables. The equality (2.2) implies that $\mathcal{T}$ is mapped isomorphically onto its image in this polynomial ring. Let $h(t):=\operatorname{dim}_{K}[\mathcal{T}]_{t}$ and $r:=\min \left\{t \geq 0 \mid[\mathcal{T}]_{t} \neq 0\right\}$. It follows that $h(t)$ is at least the number of monomials of degree $t-r$; in other words,

$$
h(t) \geq\left(\begin{array}{c}
t-r+n-1 \\
n-1
\end{array}\right) .
$$

Now consider a presentation $A \simeq R / I$ as given by assumption. Further write $m:=\mu\left(I+\mathfrak{n}^{3} / \mathfrak{n}^{3}\right)$ and $\left(A^{\prime}, \mathfrak{m}^{\prime}\right):=\left(A / \mathfrak{m}^{2}, \mathfrak{m} / \mathfrak{m}^{2}\right)$. The usual $A$-free presentation of $\Omega_{A / k}$ by means of the transpose of a Jacobian matrix induces a presentation

$$
A^{\prime m} \longrightarrow A^{\prime n} \longrightarrow A^{\prime} \otimes_{A} \Omega_{A / k} \rightarrow 0
$$

yielding, for every $t \geq 1$, an exact sequence

$$
A^{\prime m} \otimes_{A^{\prime}} \mathcal{S}_{t-1}\left(A^{\prime n}\right) \longrightarrow \mathcal{S}_{t}\left(A^{\prime n}\right) \longrightarrow A^{\prime} \otimes_{A} \mathcal{S}_{t}\left(\Omega_{A / k}\right) \rightarrow 0
$$

The equality (2.2) also says that, for every $t \geq 0$, the graded component $[\mathcal{T}]_{t}$ of $\mathcal{T}$ is a $K$-vector space of dimension $h(t)$ and a direct summand of $\mathcal{S}_{t}\left(\Omega_{A / k}\right)$ as an $A$ module. Hence $A^{\prime} \otimes_{A} \mathcal{S}_{t}\left(\Omega_{A / k}\right)$ too admits $K^{\oplus h(t)}$ as a direct summand. Therefore $\mathfrak{m}^{\prime \oplus h(t)}$ is a direct summand of the image of $A^{\prime m} \otimes_{A^{\prime}} \mathcal{S}_{t-1}\left(A^{\prime n}\right)$ in $\mathcal{S}_{t}\left(A^{\prime n}\right)$, which implies that

$$
m\left(\begin{array}{c}
t-1+n-1 \\
n-1
\end{array}\right) \geq \mu\left(\mathfrak{m}^{\prime}\right) h(t) \geq \mu\left(\mathfrak{m}^{\prime}\right)\left(\begin{array}{c}
t-r+n-1 \\
n-1
\end{array}\right)
$$

for every $t \geq 1$. Observing that $\operatorname{dim} R=\mu(\mathfrak{m})=\mu\left(\mathfrak{m}^{\prime}\right)$, this inequality contradicts the assumption $m \leq \operatorname{dim} R-1$.

Corollary 2.2. Let $(R, \mathfrak{n})$ be a regular local ring essentially of finite type over a perfect field $k$, and let $I \subset R$ be an ideal such that $A=R / I$ is reduced. Assume that one of the following conditions holds:

(i) $\mu\left(I_{\mathfrak{p}}\right) \leq \operatorname{dim} R_{\mathfrak{p}}-1$ for every non-minimal $\mathfrak{p} \in V(I)$;

(ii) $I \subset \mathfrak{n}^{3}$ and $A$ is an isolated singularity.

Then $\mathbb{S}_{A / k}$ is reduced (if and) only if it is A-torsionfree. 
Remark 2.3. The full force of Theorem 2.1 takes form in the case of a homogeneous ideal $I \subset k[\mathbf{X}]=k\left[X_{1}, \ldots, X_{n}\right]$, with $k$ a perfect field, such that $A=k[\mathbf{X}] / I$ is reduced and regular on the punctured spectrum. If $\mathbb{S}_{A / k}$ is reduced, but not torsionfree, then $\operatorname{Proj}(k[\mathbf{X}] / I) \subset \mathbb{P}_{k}^{n-1}$ lies on the intersection of $n$ independent quadrics because in this situation $\operatorname{dim}_{k}[I]_{2} \geq n$ by Theorem 2.1. There is a converse when $I \subset(\mathbf{X})^{2}$ is equidimensional and $\operatorname{dim}_{k}[I]_{2} \leq 2$ height $I$. Namely, if $\mathbb{S}_{A / k}$ is torsionfree, then $n \geq 2$ height $I+1$ (cf. the beginning of this section), and hence $\operatorname{Proj}(k[\mathbf{X}] / I) \subset \mathbb{P}_{k}^{n-1}$ cannot lie on the intersection of $n$ independent quadrics.

The next two examples illustrate the above results. The first shows that Theorem 2.1 is sharp, i.e., that the assumption on the numbers of generators cannot be relaxed. For a geometric insight it may be convenient to recall the interpretation, given in [19, 5.17], of the tangential variety to $\operatorname{Proj}(A) \subset \mathbb{P}_{k}^{n-1}$ as $\operatorname{Proj}\left(k \otimes_{A} \mathbb{R}_{A / k}\right)$.

Example 2.4. Consider the homogeneous coordinate ring $A$ of the Veronese surface in $\mathbb{P}_{k}^{5}$. Thus $A=k[\mathbf{X}] / I_{2}(\mathbf{X})$, where $\mathbf{X}$ is a symmetric $3 \times 3$ matrix of indeterminates over $k$ (assumed to be of characteristic $\neq 2$ ). Here $A$ is a CohenMacaulay domain with an isolated singularity, $I_{2}(\mathbf{X})$ is generated by quadrics, and $\mu\left(I_{2}(\mathbf{X})\right)=6=\operatorname{dim} k[\mathbf{X}]$. It can be seen with the aid of Macaulay that $\mathbb{S}_{A / k}$ is reduced. However, it is not $A$-torsionfree because $\Omega_{A / k}$ fails to have the expected number of generators locally at the irrelevant ideal $A_{+}$(see the beginning of this section). Alternatively, using the interpretation mentioned above, one could argue that the defining equation of the tangential variety (which coincides with the secant variety, hence is defined by the determinant of $\mathbf{X}$ ), when read in the presentation variables of $\mathbb{S}_{A / k}$, is a non-zero torsion element. Actually, as in the reduction step of the proof of Theorem 2.1, $\mathbb{S}_{A / k}$ has exactly two minimal primes, $\mathcal{T}$ and $(\mathbf{X}) \mathbb{S}_{A / k}$, the first of which defines $\mathbb{R}_{A / k}$.

Another computation with Macaulay shows that $\mathbb{S}_{A / k}$ is Cohen-Macaulay but $\mathcal{S}\left(\mathbb{D}_{A / k}\right)$ is not. This example incidentally answers a question posed in [10, Remark on p. 142 ] concerning the existence of a Cohen-Macaulay generic complete intersection ideal $\mathbf{D} \subset S$, with $S$ and $S / \mathbf{D}$ Cohen-Macaulay, for which the symmetric algebra $\mathcal{S}\left(\mathbf{D} / \mathbf{D}^{2}\right)$ is Cohen-Macaulay but the symmetric algebra $\mathcal{S}(\mathbf{D})$ is not. Mark Johnson obtained examples with the latter behavior even in a polynomial ring ([15, Example 1]).

Example 2.5. Let $A=k[\mathbf{X}] / I_{2}(\mathbf{X})$, where $(\mathbf{X})$ is the $r$-catalecticant matrix

$$
\left(\begin{array}{cccc}
X_{1} & X_{2} & X_{3} & X_{4} \\
X_{r+1} & X_{r+2} & X_{r+3} & X_{r+4}
\end{array}\right)
$$

with $1 \leq r \leq 4$. Note that for $r=4$ we obtain the $2 \times 4$ generic matrix and for $r=1$ the usual $2 \times 4$ generic Hankel matrix. Also $A$ is a Cohen-Macaulay (domain) in all cases, being a specialization of the generic situation. Clearly $A$ is an isolated singularity. For values $1 \leq r \leq 2$ the Zariski tangent algebra $\mathbb{S}_{A / k}$ is neither Cohen-Macaulay nor torsionfree. Geometrically, we see the reason for $\mathbb{S}_{A / k} \neq \mathbb{R}_{A / k}$ since in this range the tangential variety Proj $\left(k \otimes_{A} \mathbb{R}_{A / k}\right) \subset \mathbb{P}_{k}^{r+3}$ to $\operatorname{Proj}\left(k[\mathbf{X}] / I_{2}(\mathbf{X})\right) \subset \mathbb{P}_{k}^{r+3}$ is a proper subvariety of the ambient space. For values $3 \leq r \leq 4$ the Zariski tangent algebra $\mathbb{S}_{A / k}$ is Cohen-Macaulay (a computer calculation) and hence torsionfree because $r+4 \geq 7=2$ height $I_{2}(\mathbf{X})+1$ (see [24, $3.3])$. 
2.2. Reducedness: An analogue of Berger's conjecture. When $\operatorname{dim} A=1$ one can make Theorem 2.1 more precise. Here it is natural to work in the more general setting of complete $k$-algebras. Instead of the module of Kähler differentials we use the universally finite module of differentials, defined in an analogous way as $\mathbb{D} / \mathbb{D}^{2}$, where $\mathbb{D}$ now is the kernel of the multiplication map $A \widehat{\otimes}_{k} A \longrightarrow A$. Our result is reminiscent of Berger's well-known conjecture asserting that the module of differentials of a reduced curve singularity over a perfect field cannot be torsionfree (see [3]). It is clear at least that the entire symmetric algebra $\mathbb{S}_{A / k}$ cannot be $A$ torsionfree, as otherwise the module of differentials would satisfy condition $\left(F_{1}\right)$, which translates into the inequality $\operatorname{edim} A \leq 2 \operatorname{dim} A-1=1$ (see the discussion at the beginning of Section 2).

Theorem 2.6. Let $k$ be an algebraically closed field and let $A=k\left[\left[x_{1}, \ldots, x_{n}\right]\right]$ be a one-dimensional domain. Then $\mathbb{S}_{A / k}$ is reduced (if and) only if $A$ is regular.

Proof. Assume that $\mathbb{S}_{A / k}$ is reduced. To argue by way of contradiction assume that $n=\operatorname{edim} A \geq 2$. Now, the integral closure of $A$ is the power series ring $k[[t]]$ in one variable. Letting $v(-)$ denote the $t$-adic valuation, we may arrange so that

$$
v\left(x_{1}\right)<v\left(x_{2}\right)<v\left(x_{3}\right) \leq \cdots \leq v\left(x_{n}\right)
$$

and $v\left(x_{2}\right) / v\left(x_{1}\right)$ is not an integer (the condition on $v\left(x_{j}\right)$, for $j \geq 3$, being vacuous if $n=2)$. Write $A=k\left[\left[X_{1}, \ldots, X_{n}\right]\right] /\left(f_{1}, \ldots, f_{m}\right)$ with $f_{i} \in\left(X_{1}, \ldots, X_{n}\right)^{2}, T_{j}=d X_{j}$ and $t_{j}=d x_{j}$, where $d$ denotes the universally finite derivation. Notice that

$$
\mathbb{S}_{A / k} \simeq A\left[t_{1}, \ldots, t_{n}\right]=A\left[T_{1}, \ldots, T_{n}\right] /\left(\sum_{j=1}^{n} \frac{\partial f_{i}}{\partial x_{j}} T_{j} \mid 1 \leq i \leq m\right) .
$$

Here $\partial f_{i} / \partial x_{j}$ denotes the image of $\partial f_{i} / \partial X_{j}$ in $A$. Clearly these elements are contained in the maximal ideal $\mathfrak{m}$ of $A$.

As before, let $\mathcal{T}$ denote the $A$-torsion of $\mathbb{S}_{A / k}$. By the reducedness of $\mathbb{S}_{A / k}$, one has $\mathcal{T} \cap \mathfrak{m S}_{A / k}=0$ as in (2.2) - note that the localization reduction step there is needless in the present context. In particular $\operatorname{Supp}\left(\mathfrak{m} \mathbb{S}_{A / k}\right) \cap V\left(\mathfrak{m} \mathbb{S}_{A / k}\right) \subset$ $V\left(\mathcal{T}+\mathfrak{m} \mathbb{S}_{A / k}\right)$ as subsets of $\operatorname{Spec}\left(\mathbb{S}_{A / k}\right)$. On the other hand,

$$
\operatorname{dim} \mathbb{S}_{A / k} /\left(\mathcal{T}+\mathfrak{m} \mathbb{S}_{A / k}\right)=\operatorname{dim} \mathbb{R}_{A / k} / \mathfrak{m} \mathbb{R}_{A / k}=1 .
$$

Since $\left(\mathfrak{m} \mathbb{S}_{A / k}, t_{3}, \ldots, t_{n}\right)$ is a two-dimensional prime ideal of $\mathbb{S}_{A / k}$, it follows that $\left(\mathfrak{m} \mathbb{S}_{A / k}, t_{3}, \ldots, t_{n}\right) \notin \operatorname{Supp}\left(\mathfrak{m} \mathbb{S}_{A / k}\right)$.

Thus $\left(\mathfrak{m} \mathbb{S}_{A / k}\right)_{\left(\mathfrak{m} \mathbb{S}_{A / k}, t_{3}, \ldots, t_{n}\right)}=0$. From the presentation

$$
\mathbb{S}_{A / k} /\left(t_{3}, \ldots, t_{n}\right)=A\left[T_{1}, T_{2}\right] / \mathcal{J}
$$

with

we see that

$$
\mathcal{J}=\left(\frac{\partial f_{i}}{\partial x_{1}} T_{1}+\frac{\partial f_{i}}{\partial x_{2}} T_{2} \mid 1 \leq i \leq m\right)
$$

$$
\mathfrak{m} A\left[T_{1}, T_{2}\right]_{\mathfrak{m} A\left[T_{1}, T_{2}\right]}=\mathcal{J} A\left[T_{1}, T_{2}\right]_{\mathfrak{m} A\left[T_{1}, T_{2}\right]} .
$$

In particular, there exists a polynomial $g\left(T_{1}, T_{2}\right) \in A\left[T_{1}, T_{2}\right] \backslash \mathfrak{m} A\left[T_{1}, T_{2}\right]$ with

$$
g\left(T_{1}, T_{2}\right) \cdot \mathfrak{m} \subset\left(\frac{\partial f_{i}}{\partial x_{1}} T_{1}+\frac{\partial f_{i}}{\partial x_{2}} T_{2} \mid 1 \leq i \leq m\right) .
$$


Comparing coefficients, we conclude that

$$
\mathfrak{m}=\left(\frac{\partial f_{i}}{\partial x_{1}}, \frac{\partial f_{i}}{\partial x_{2}} \mid 1 \leq i \leq m\right) .
$$

Since $v\left(x_{1}\right)$ is the smallest positive element in the value semigroup of $A$, there exists an $i$ such that $v\left(x_{1}\right)=v\left(\partial f_{i} / \partial x_{1}\right)$ or $v\left(x_{1}\right)=v\left(\partial f_{i} / \partial x_{2}\right)$. Set $f:=$ $f_{i}$. Assuming the first, we write $f=a X_{1}^{2}+g$, with $a \in k$ and $g \in\left(X_{1}^{3}\right)+$ $\left(X_{1}, \ldots, X_{n}\right)\left(X_{2}, \ldots, X_{n}\right)$. Obviously $v\left(g\left(x_{1}, \ldots, x_{n}\right)\right)>2 v\left(x_{1}\right)$, hence $v\left(\partial g / \partial x_{1}\right)$ $>v\left(x_{1}\right)$. Therefore $v\left(\partial f / \partial x_{1}\right)=v\left(x_{1}\right)$ implies $a \neq 0$, while $f\left(x_{1}, \ldots, x_{n}\right)=0$ implies $a=0$. This is a contradiction.

Next assume $v\left(x_{1}\right)=v\left(\partial f / \partial x_{2}\right)$ and write

$$
f=\sum_{i=2}^{\infty} a_{i} X_{1}{ }^{i}+b X_{1} X_{2}+h
$$

where $a_{i} \in k, b \in k$ and $h \in\left(X_{1}^{2}, X_{2}\right) X_{2}+\left(X_{1}, \ldots, X_{n}\right)\left(X_{3}, \ldots, X_{n}\right)$. Here $v\left(h\left(x_{1}, \ldots, x_{n}\right)\right)>v\left(x_{1}\right)+v\left(x_{2}\right)$, hence $v\left(\partial h / \partial x_{2}\right)>v\left(x_{1}\right)$. Therefore $v\left(\partial f / \partial x_{2}\right)=$ $v\left(x_{1}\right)$ implies $b \neq 0$. But then $f\left(x_{1}, \ldots, x_{n}\right)=0$ implies

$$
v\left(x_{1}\right)+v\left(x_{2}\right)=v\left(b x_{1} x_{2}\right)=v\left(\sum_{i=2}^{\infty} a_{i} x_{1}^{i}\right)=\operatorname{lv}\left(x_{1}\right)
$$

for some integer $l$. But this contradicts our assumption that $v\left(x_{2}\right) / v\left(x_{1}\right)$ is not an integer.

Remark 2.7. The above result means that the first jet scheme of an integral algebroid curve singularity over an algebraically closed field cannot be reduced.

The proof of Theorem 2.6 gives the following stronger result:

Theorem 2.8. Let $k$ be an algebraically closed field, let $A=k\left[\left[x_{1}, \ldots, x_{n}\right]\right]$ be a one-dimensional ring and assume that $\mathbb{S}_{A / k}$ is reduced. Then $A / \mathfrak{p}$ is regular for every minimal prime ideal $\mathfrak{p}$ of $A$. If in addition $\operatorname{char}(k)=0$ and $A$ is quasihomogeneous (i.e., the completion of a positively graded $k$-algebra), then $A$ is regular.

Proof. We use the notation of the previous proof. Of course $A$ is reduced. Suppose there exists a minimal prime $\mathfrak{p}$ of $A$ such that $A / \mathfrak{p}$ is not regular. Then choose a minimal generating set $x_{1}, \ldots, x_{n}$ of $\mathfrak{m} / \mathfrak{p}$ such that

$$
v\left(x_{1}+\mathfrak{p}\right)<v\left(x_{2}+\mathfrak{p}\right)<v\left(x_{3}+\mathfrak{p}\right) \leq \cdots \leq v\left(x_{n}+\mathfrak{p}\right)
$$

and the ratio of the first two values is not an integer (here $v$ is the $t$-adic valuation on the integral closure $k[[t]]$ of $A / \mathfrak{p})$. The rest of the proof proceeds as before, replacing $\mathbb{S}_{A / k} /\left(t_{3}, \ldots, t_{n}\right)$ by $\mathbb{S}_{A / k} /\left(\mathfrak{p}, t_{3}, \ldots, t_{n}\right)$.

Now assume that $\operatorname{char}(k)=0$ and $A$ is quasi-homogeneous. Let $\tau$ denote the kernel of the Euler map $\Omega_{A / k} \rightarrow \mathfrak{m}$. Since $\tau$ is the torsion of $\Omega_{A / k}$, by the reducedness of $\mathbb{S}_{A / k}$ we have $\tau \cap \mathfrak{m} \Omega_{A / k}=0$ as in (2.2); hence $\tau$ is a direct summand of $\Omega_{A / k}$. It follows that $\tau=0$ since $\mu\left(\Omega_{A / k}\right)=\operatorname{edim} R=\mu(\mathfrak{m})$. But then $A$ is regular because in the quasi-homogeneous case the Berger conjecture is true (see [18, 4.4]). 
2.3. Normality versus reflexivity. Let $A$ be a normal domain essentially of finite type over a perfect field $k$. An interesting graded object related to $\mathbb{S}_{A / k}$ is the "reflexive closure" $\mathbb{B}_{A / k}:=\mathcal{B}_{A}\left(\Omega_{A / k}\right)=\bigoplus_{i>0}\left(\Omega_{A / k}^{i}\right)^{* *}$ of $\mathbb{R}_{A / k}=\bigoplus_{i \geq 0} \Omega_{A / k}^{i}$, where $-{ }^{*}$ stands for $A$-duals. The reflexive closure is again an algebra, as can be seen by localizing at the height one prime ideals of $R$ (see also [28, p. 160]). There is a natural $A$-algebra inclusion $\mathbb{R}_{A / k} \subset \mathbb{B}_{A / k}$ (see Subsection 3.2, where we study cases when this inclusion is an equality). Now, if $\mathbb{S}_{A / k}$ is normal, then it is torsionfree, so $\mathbb{S}_{A / k}=\mathbb{R}_{A / k} \subset \mathbb{B}_{A / k}$. In this part we give sufficient conditions in order that, in the presence of the normality of $\mathbb{S}_{A / k}$, the inclusion $\mathbb{S}_{A / k} \subset \mathbb{B}_{A / k}$ be an equality. The conditions are stated, once again, in terms of the number of "quadrics" contained in $I$, when $I$ is the defining ideal of $A$ in a regular local ring $(R, \mathfrak{n})$ essentially of finite type over $k$.

First we prove a more general result.

Proposition 2.9. Let $(R, \mathfrak{n})$ be a regular local ring essentially of finite type over a perfect field $k$ and let $I \subset \mathfrak{n}^{2}$ be an ideal such that $A=R / I$ is reduced. Write $g$ for the height of $I$ and $\mathfrak{m}$ for the maximal ideal of $A$.

(a) If $\mathbb{S}_{A / k}$ is equidimensional and $\left(\mathbb{S}_{A / k}\right)_{\mathfrak{m} \mathbb{S}_{A / k}}$ is regular, then $\mu\left(I+\mathfrak{n}^{3} / \mathfrak{n}^{3}\right) \geq$ $2 g$.

(b) Assume that char $(k)=0$ and $R$ is the localization of the polynomial ring $k\left[X_{1}, \ldots, X_{n}\right]$ at its homogeneous maximal ideal. Let $I_{2}$ denote the ideal of $R$ generated by 2 -forms in $X_{1}, \ldots, X_{n}$ such that $I+\mathfrak{n}^{3}=I_{2}+\mathfrak{n}^{3}$. If $\mathbb{S}_{A / k}$ is equidimensional and $\left(\mathbb{S}_{A / k}\right)_{\mathfrak{m} \mathbb{S}_{A / k}}$ is regular, then $\ell\left(I_{2}\right)=2 g$.

Proof. Knowingly, $\mathbb{S}_{R / k}$ is a polynomial ring over $R$. In a more precise way, let $d: R \longrightarrow \Omega_{R / k}$ stand for the universal derivation. Then $\mathbb{S}_{R / k} \simeq R[\mathbf{T}]$, where $\mathbf{T}=T_{1}, \ldots, T_{n}$ are variables that may be chosen to be the differentials (i.e., the images under $d$ ) of elements in $R$; in part (b) they may be chosen as the differentials $d X_{1}, \ldots, d X_{n}$. Set $K:=R / \mathfrak{n}$, so that the residue field of $R(\mathbf{T})=R[\mathbf{T}]_{\mathfrak{n} R[\mathbf{T}]}$ is $K(\mathbf{T})$. Write $r:=\operatorname{trdeg}_{k}(A / \mathfrak{m})=\operatorname{trdeg}_{k} K$. There is a presentation

$$
\left(\mathbb{S}_{A / k}\right)_{\mathfrak{m} \mathbb{S}_{A / k}} \simeq R(\mathbf{T}) / \mathcal{J}
$$

Let $\mathfrak{p}$ be a minimal prime of $A$ with $\operatorname{dim} A / \mathfrak{p}=\operatorname{dim} A$. Since $\mathbb{S}_{A / k}$ is equidimensional, one has $\operatorname{dim} \mathbb{S}_{A / k}=\operatorname{dim} A / \mathfrak{p}+\operatorname{rank} \Omega_{A_{\mathfrak{p}} / k}$ (see [25, the proof of 1.1.1]). Therefore, by (2.1) one gets

$\operatorname{dim} \mathbb{S}_{A / k}=\operatorname{dim} A / \mathfrak{p}+\operatorname{trdeg}_{k}(A / \mathfrak{p})=2 \operatorname{dim} A / \mathfrak{p}+r=2(n-r-g)+r=2 n-2 g-r$.

Hence, again by the equidimensionality of $\mathbb{S}_{A / k}$,

height $\mathcal{J}=\operatorname{dim} R(\mathbf{T})-$ height $\mathbb{S}_{A / k}=n-r-\left(\operatorname{dim} \mathbb{S}_{A / k}-\operatorname{dim} \mathbb{S}_{A / k} / \mathfrak{m} \mathbb{S}_{A / k}\right)$ $=n-r-\left(\operatorname{dim} \mathbb{S}_{A / k}-n\right)=n-r-((2 n-2 g-r)-n)=2 g$.

Thus, since $\left(\mathbb{S}_{A / k}\right)_{\mathfrak{m} \mathbb{S}_{A / k}}$ is regular, we have

$$
\operatorname{dim}_{K(\mathbf{T})}\left(\mathcal{J}+\mathcal{N}^{2} / \mathcal{N}^{2}\right)=2 g,
$$

where $\mathcal{N}=\mathfrak{n} R(\mathbf{T})$.

Now let $f_{1}, \ldots, f_{m}$ in $R$ be so chosen that they generate $I$ modulo $\mathfrak{n}^{3}$ for part (a) and that they be homogeneous quadrics in $k\left[X_{1}, \ldots, X_{n}\right]$ generating the ideal 
$I_{2}$ for part (b). In either case, one has

$$
\mathcal{J}+\mathcal{N}^{2} / \mathcal{N}^{2}=\left(d f_{i} \mid 1 \leq i \leq m\right)+\mathcal{N}^{2} / \mathcal{N}^{2} .
$$

It follows that $m \geq 2 g$, thus proving (a).

To prove (b), let $f:=f(\mathbf{X})$ denote any of the chosen 2 -forms $f_{1}, \ldots, f_{m}$ in $k[\mathbf{X}]=k\left[X_{1}, \ldots, X_{n}\right]$. Since the Hessian matrix $\varphi$ of $f$ is symmetric, one has

$$
\mathbf{X} \cdot \varphi \cdot \mathbf{T}^{t}=\mathbf{T} \cdot \varphi \cdot \mathbf{X}^{t}
$$

As the Hessians of $f(\mathbf{X})$ and of $f(\mathbf{T})$ coincide, this shows that

$$
\sum_{j=1}^{n} \frac{\partial f(\mathbf{X})}{\partial X_{j}} T_{j}=\sum_{j=1}^{n} \frac{\partial f(\mathbf{T})}{\partial T_{j}} X_{j}
$$

hence

$$
d f=\sum_{j=1}^{n} \frac{\partial f(\mathbf{T})}{\partial T_{j}} X_{j} .
$$

We deduce that

$$
\begin{aligned}
\operatorname{dim}_{k(\mathbf{T})}\left(\left(d f_{i} \mid 1 \leq i \leq m\right)+\mathcal{N}^{2} / \mathcal{N}^{2}\right) & =\operatorname{rank}\left(\frac{\partial f_{i}(\mathbf{T})}{\partial T_{j}}\right)_{i, j}=\operatorname{rank}\left(\frac{\partial f_{i}(\mathbf{X})}{\partial X_{j}}\right)_{i, j} \\
& =n-\operatorname{rank} \Omega_{R / k\left[f_{1}, \ldots, f_{m}\right]} \\
& =n-\operatorname{trdeg}_{k\left[f_{1}, \ldots, f_{m}\right]} R \\
& =\operatorname{trdeg}_{k} k\left[f_{1}, \ldots, f_{m}\right]=\operatorname{dim} k\left[f_{1}, \ldots, f_{m}\right]
\end{aligned}
$$

(see also [20,1.1]). On the other hand, since $f_{1}, \ldots, f_{m}$ are forms of the same degree generating $I_{2}$, we have $k\left[f_{1}, \ldots, f_{m}\right] \simeq k \otimes_{R} \mathcal{R}\left(I_{2}\right)$, hence $\operatorname{dim} k\left[f_{1}, \ldots, f_{m}\right]=\ell\left(I_{2}\right)$. Summing up, we have proved that

$$
2 g=\operatorname{dim}_{k(\mathbf{T})}\left(\mathcal{J}+\mathcal{N}^{2} / \mathcal{N}^{2}\right)=\ell\left(I_{2}\right) .
$$

Theorem 2.10. Let $A$ be a normal domain essentially of finite type over a perfect field $k$. Assume that one of the following conditions holds:

(i) For every non-regular prime $\mathfrak{p} \in \operatorname{Spec}(A)$ there is a presentation $A_{\mathfrak{p}} \simeq R / I$, where $(R, \mathfrak{n})$ is a regular local ring essentially of finite type over $k$ and $I \subset \mathfrak{n}^{2}$ is an ideal satisfying $\mu\left(I+\mathfrak{n}^{3} / \mathfrak{n}^{3}\right) \leq 2$ height $I-1$;

(ii) $(\operatorname{char}(k)=0) A$ is an isolated singularity and has a presentation $A \simeq$ $R / I$ with $(R, \mathfrak{n})$ the localization of $k\left[X_{1}, \ldots, X_{n}\right]$ at $\left(X_{1}, \ldots, X_{n}\right)$, such that $\ell\left(I_{2}\right) \neq 2$ height $I$; here $I_{2}$ denotes the ideal of $R$ generated by 2 -forms in $X_{1}, \ldots, X_{n}$ such that $I+\mathfrak{n}^{3}=I_{2}+\mathfrak{n}^{3}$.

Then $\mathbb{S}_{A / k}$ is normal (if and) only if $\mathbb{S}_{A / k}=\mathbb{B}_{A / k}$.

Proof. Assume that $\mathbb{S}_{A / k}$ is normal. In this case $\mathbb{S}_{A / k}$ is a domain, since $\operatorname{Spec}\left(\mathbb{S}_{A / k}\right)$ is connected. We prove that the natural inclusion $\mathbb{S}_{A / k} \subset \mathbb{B}_{A / k}$ is an equality. It suffices to show this after localizing at the height one primes of $\mathbb{S}_{A / k}$. Thus, let $\mathfrak{q} \in \operatorname{Spec}\left(\mathbb{S}_{A / k}\right)$ be a prime of height one. We are done once we prove that $A_{\mathfrak{q} \cap A}$ is regular. Now, replacing $A$ by $A_{\mathfrak{q} \cap A}$, we may assume that $(A, \mathfrak{m})$ is local and $\mathfrak{m} \subset \mathfrak{q}$. By assumption $\left(\mathbb{S}_{A / k}\right)_{\mathfrak{q}}$ is regular, hence so is its further localization $\left(\mathbb{S}_{A / k}\right)_{\mathfrak{m} \mathbb{S}_{A / k}}$. According to Proposition 2.9 this contradicts our assumptions, unless $A$ is regular. 
Corollary 2.11. Let $(R, \mathfrak{n})$ be a regular local ring essentially of finite type over a perfect field $k$ and let $I \subset \mathfrak{n}^{2}$ be an ideal such that $A=R / I$ is an equidimensional isolated singularity. If $\mathbb{S}_{A / k}$ is reduced and $\left(\mathbb{S}_{A / k}\right)_{\mathfrak{m} \mathbb{S}_{A / k}}$ is regular, then

$$
\mu\left(I+\mathfrak{n}^{3} / \mathfrak{n}^{3}\right) \geq \min \{\operatorname{dim} R, 2 \text { height } I\} .
$$

Proof. If $\mu\left(I+\mathfrak{n}^{3} / \mathfrak{n}^{3}\right) \leq \operatorname{dim} R-1$, then $\mathbb{S}_{A / k}$ is torsionfree by Theorem 2.1. Since $A$ is reduced and equidimensional, it follows that $\mathbb{S}_{A / k}$ is equidimensional as well (see (2.1) and [25, the proof of 1.1.1]). Therefore $\mu\left(I+\mathfrak{n}^{3} / \mathfrak{n}^{3}\right) \geq 2$ height $I$ by Proposition 2.9(a).

\section{The Rees algebra of the module of Differentials}

3.1. Cohen-Macaulayness of $\mathbb{R}_{A / k}$. Let $A$ be a local Cohen-Macaulay ring and let $E$ be a finite $A$-module of projective dimension at most one. If $E$ satisfies condition $\left(F_{1}\right)$, then the symmetric algebra $\mathcal{S}_{A}(E)$ is a Cohen-Macaulay torsionfree $A$-algebra (see [1, Propositions 3 and 4], [13, 1.1], [24, 3.4]); in particular, the Rees algebra $\mathcal{R}_{A}(E)$ is Cohen-Macaulay. The question as to whether, conversely, $\mathcal{R}_{A}(E)$ being Cohen-Macaulay implies $\left(F_{1}\right)$ has a negative answer in general even if $E$ satisfies $\left(F_{0}\right)$ (see $\left.[23,4.7]\right)$. The theorem below will show that this converse holds when $E$ is the module of differentials of a complete intersection over a field of characteristic zero. This shows that Cohen-Macaulayness is a rather restrictive property for $\mathbb{R}_{A / k}$.

Theorem 3.1. Let $k$ be a field of characteristic zero and let $A$ be a $k$-algebra essentially of finite type which is locally a complete intersection. Assume the following conditions:

(i) $\operatorname{edim} A_{\mathfrak{p}} \leq 2 \operatorname{dim} A_{\mathfrak{p}}$ for every prime $\mathfrak{p} \in \operatorname{Spec}(A)$;

(ii) $\mathbb{R}_{A / k}$ is Cohen-Macaulay.

Then $\operatorname{edim} A_{\mathfrak{p}} \leq 2 \operatorname{dim} A_{\mathfrak{p}}-1$ for every non-minimal prime $\mathfrak{p} \in \operatorname{Spec}(A)$.

Proof. Arguing by way of contradiction, let $\mathfrak{p} \in \operatorname{Spec}(A)$ be minimal such that $\operatorname{edim} A_{\mathfrak{p}} \geq 2 \operatorname{dim} A_{\mathfrak{p}} \geq 2$. By localizing at this prime, we may assume that $(A, \mathfrak{m})$ is local and, by (i), that $\operatorname{edim} A=2 \operatorname{dim} A$.

Next we reduce to the case where the residue field $K:=A / \mathfrak{m}$ is algebraic over $k$. To do this, let $r$ be the transcendence degree of $K$ over $k$ and suppose $r \geq 1$. Write $A=k\left[x_{1}, \ldots, x_{m}\right]_{\mathfrak{p}}$ and pick $r$ general $k$-linear combinations $y_{1}, \ldots, y_{r}$ of $x_{1}, \ldots, x_{m}$ such that, in particular, their residues yield a transcendence basis of $K$ over $k$. Notice that $L:=k\left(y_{1}, \ldots, y_{r}\right)$ is a subfield of $A$. Furthermore, $\Omega_{A / L}=$ $\Omega_{A / k} /\left(A d y_{1}+\cdots+A d y_{r}\right)$ with $d: A \longrightarrow \Omega_{A / k}$ denoting the universal derivation. On the other hand, rank $\Omega_{A / k}>r$ according to (2.1). Hence the general choice of $y_{1}, \ldots, y_{r}$ and the Cohen-Macaulayness of $\mathbb{R}_{A / k}$ yield an isomorphism

$$
\mathcal{R}\left(\Omega_{A / L}\right) \simeq \mathcal{R}\left(\Omega_{A / k}\right) /\left(d y_{1}, \ldots, d y_{r}\right),
$$

which shows in particular that this Rees algebra is again Cohen-Macaulay (see [6, $2.2(\mathrm{f})]$ ). Thus, replacing $k$ by $L$, we may assume henceforth that $K$ is algebraic over $k$.

Set $d:=\operatorname{dim} A$ (a number, not the universal derivation!) and induct on $d$. If $d=1$, the Cohen-Macaulayness of $\mathbb{R}_{A / k}$ implies that $\Omega_{A / k}$ modulo torsion is free (see [23, 4.3]), which would force $A$ to be regular (see [16, Theorem]), thus contradicting the equality $\operatorname{edim} A=2$. 
Hence we may suppose that $d \geq 2$. Write $A=R / I$, where $(R, \mathfrak{n})$ is a regular local ring essentially of finite type over $k$ and $I \subset \mathfrak{n}^{2}$ is an ideal. Set $n:=\operatorname{dim} R=$ $\operatorname{edim} A=2 d$ and let $\delta: R \longrightarrow \Omega_{R / k}$ be the universal derivation. Since $R$ is smooth over $k$ and the extension $k \subset K$ is separable algebraic, there exist $n$ elements $X_{1}, \ldots, X_{n}$ in $\mathfrak{n}$ so that $\delta X_{1}, \ldots, \delta X_{n}$ form a basis of $\Omega_{R / k}$. For an element $f$ in $R$ we can then define the "partial derivatives" $\frac{\partial f}{\partial X_{i}}$ via the equation $\delta f=\sum \frac{\partial f}{\partial X_{i}} d X_{i}$, and we write $\frac{\partial f}{\partial x_{i}}$ for their images in $A$. By assumption, $I$ is generated by an $R$-regular sequence $f_{1}, \ldots, f_{d}$. We consider the $n$ by $d$ matrices

$$
\Theta=\left(\frac{\partial f_{j}}{\partial X_{i}}\right) \quad \text { and } \quad \theta=\left(\frac{\partial f_{j}}{\partial x_{i}}\right) .
$$

Now $\theta$ presents the $A$-module $\Omega_{A / k}$, which has projective dimension one and satisfies condition $\left(F_{1}\right)$ locally in codimension $d-1 \geq 1$ by the inductive hypothesis. The analytic spread of this module is at most $\operatorname{dim} A+\operatorname{rank} \Omega_{A / k}-1$ (see [23, 2.3]), and the latter number is $2 d-1=n-1$ (see (2.1)). Since $\mathbb{R}_{A / k}$ is Cohen-Macaulay, we may then apply [23, 2.3, 4.7(a) and 4.8] with $s=n-1$ to conclude that after a $k$-linear change of basis,

$$
I_{1}(\theta)=\left(\frac{\partial f_{1}}{\partial x_{n}}, \ldots, \frac{\partial f_{d}}{\partial x_{n}}\right)
$$

Thus

$$
I_{1}(\Theta) \subset\left(\frac{\partial f_{1}}{\partial X_{n}}, \ldots, \frac{\partial f_{d}}{\partial X_{n}}, I\right) .
$$

Set $J:=I_{1}(\Theta)$ and $J_{0}:=\left(\frac{\partial f_{1}}{\partial X_{n}}, \ldots, \frac{\partial f_{d}}{\partial X_{n}}\right)$, and notice that $J+I=J_{0}+I$ by the above. Furthermore height $J_{0} \leq d$.

We show that

$$
I \subset \overline{\mathfrak{n} J}
$$

where $^{-}$denotes integral closure. Thus, let $f$ be an element of $\mathfrak{n}$. We argue that

$$
f \in \overline{\mathfrak{n}\left(\frac{\partial f}{\partial X_{1}}, \ldots, \frac{\partial f}{\partial X_{n}}\right)} .
$$

This is well known in slightly different settings (see, e.g., [26, 7.1.5]), but for lack of the particular reference needed here we include a proof. By the valuative criterion for integral dependence it suffices to show that $f \in \mathfrak{n}\left(\frac{\partial f}{\partial X_{1}}, \ldots, \frac{\partial f}{\partial X_{n}}\right) V$ for every local extension $R \subset V$, with $V$ a complete discrete valuation ring (see, e.g., [26, 6.8.4]). Since the extension $k \subset K$ is separable, there exists a coefficient field $K$ of $V$ that contains $k$. Clearly $V \simeq K[[t]]$. Write $D: K[[t]] \longrightarrow K[[t]] D t \simeq K[[t]]$ for the universally finite $K$-derivation of $K[[t]]$, which maps a power series $h(t)$ to $h^{\prime}(t) D t$. As $R \subset K[[t]]$ and $k \subset K$, one can restrict $D$ to $R$ and this restriction factors though $\delta$. Thus $D f=\sum \frac{\partial f}{\partial X_{i}} D X_{i}$ or, equivalently, $f^{\prime}=\sum \frac{\partial f}{\partial X_{i}} X_{i}^{\prime}$. Since the characteristic of $K$ is zero, one has $h V=t h^{\prime} V$ for every power series $h \in V$ that in not a unit in $V$. Recall that $f$ and $X_{1}, \ldots, X_{n}$ are in $\mathfrak{n}$, hence in the maximal ideal of $V$. Thus, multiplying both sides of the above equation for $f^{\prime}$ by $t$, one sees that indeed $f \in \sum \frac{\partial f}{\partial X_{i}} X_{i} V \subset \mathfrak{n}\left(\frac{\partial f}{\partial X_{1}}, \ldots, \frac{\partial f}{\partial X_{n}}\right) V$.

Having shown the inclusion $I \subset \overline{\mathfrak{n} J}$, we conclude that

$$
J+I=J_{0}+I \subset J_{0}+\overline{\mathfrak{n} J} \subset \overline{J_{0}+\mathfrak{n}(J+I)} .
$$


The valuative criterion for integral dependence now yields $J+I \subset \overline{J_{0}}$. Therefore height $(J+I)=$ height $J_{0} \leq d$. Hence $\operatorname{dim} R /(J+I) \geq 2 d-d=d \geq 2$. But this is impossible because $R /(J+I) \simeq A / I_{1}(\theta)$ has dimension zero by assumption (i).

Remark 3.2. The theorem implies the following geometric result: let $X \subset \mathbb{P}_{k}^{2 d+1}$ denote a $d$-dimensional smooth complete intersection over a field $k$ of characteristic zero, with homogeneous coordinate ring $A$. If $\mathbb{R}_{A / k}$ is Cohen-Macaulay, then $X$ is degenerate.

Question 3.3. It seems reasonable to ask whether Theorem 3.1 holds without assumption (i).

3.2. Normality of $\mathbb{R}_{A / k}$. Let $A$ be a normal domain and let $E$ be a finite $A$ module of rank $r$. We write $E^{i}$ for the $i$ th graded component $\mathcal{R}_{A}(E)_{i}$ of $\mathcal{R}_{A}(E)$ and call it the $i$ th power of $E$. Notice that $E^{i}$ is also the $i$ th symmetric power of $E$ modulo $A$-torsion, $E^{i}=\mathcal{S}_{i}(E) / \tau_{A}\left(\mathcal{S}_{i}(E)\right)$. Since $E$ has rank $r, E^{1}$ can be embedded into $F:=A^{r}$ and any such embedding induces an embedding of $\mathcal{R}_{A}(E)$ into a polynomial ring $\mathcal{S}_{A}(F)=A[\mathbf{t}]=A\left[t_{1}, \ldots, t_{r}\right]$ as a graded $A$-subalgebra. Let $\overline{\mathcal{R}_{A}(E)}$ denote the integral closure of $\mathcal{R}_{A}(E)$ in $A[\mathbf{t}]$, which is again a graded $A$-subalgebra. Since $A$ is normal, this integral closure coincides with the integral closure of $\mathcal{R}_{A}(E)$ in its field of fractions, and in particular does not depend on the chosen embedding $E^{1} \subset F$. We denote by $\overline{E^{i}} \subset A[\mathbf{t}]_{i}$ the $i$ th graded component of $\overline{\mathcal{R}_{A}(E)}$ and call it the ith normalized power of $E$. We say that $E$ is integrally closed if $E^{1}=\overline{E^{1}}$ and that $E$ is normal if $E^{i}=\overline{E^{i}}$ for every $i \geq 1$ or, equivalently, if $\mathcal{R}_{A}(E)$ is normal. One can see that there are inclusions

$$
E^{i} \subset \overline{E^{i}} \subset\left(E^{i}\right)^{* *} \subset \mathcal{S}_{i}\left(A^{r}\right) .
$$

Recall that $\mathcal{B}_{A}(E):=\bigoplus_{i \geq 0}\left(E^{i}\right)^{* *}$; the property $\left(L_{2}\right)$ used in the next proposition has been defined in the preliminaries of Section 2 .

Proposition 3.4. Let $A$ be a universally catenary normal domain and let $E$ be a finite A-module. The following are equivalent:

(1) $\mathcal{R}_{A}(E)$ is normal, and $E$ satisfies condition $\left(L_{2}\right)$;

(2) $\mathcal{R}_{A}(E)$ satisfies condition $\left(S_{2}\right)$ of Serre, and $E$ satisfies condition $\left(L_{2}\right)$;

(3) $\mathcal{R}_{A}(E)=\mathcal{B}_{A}(E)$.

Proof. (1) $\Rightarrow(2)$ : This is obvious.

$(2) \Rightarrow(3)$ : It suffices to show that all powers of $E$ satisfy Serre's condition $\left(S_{2}\right)$. For that let $\mathfrak{p} \subset A$ be any prime ideal of height at least two. We may assume that $A$ is local with maximal ideal $\mathfrak{m}=\mathfrak{p}$. Then height $\mathfrak{m} \mathcal{R}_{A}(E)=\operatorname{dim} \mathcal{R}_{A}(E)-\ell(E)=$ $\operatorname{dim} A+\operatorname{rank} E-\ell(E) \geq 2$, the first equality by the catenarian assumption, the second one by a standard formula (see $[23,2.2]$ ), and the last inequality by $\left(L_{2}\right)$. Since $\mathcal{R}_{A}(E)$ satisfies condition $\left(S_{2}\right)$, the ideal $\mathfrak{m}$ then contains an $\mathcal{R}_{A}(E)$-regular sequence of two elements. This is also a regular sequence on each power $E^{i}$, hence depth $E^{i} \geq 2$ for every $i \geq 1$.

$(3) \Rightarrow(1)$ : By (3.1), we have equality of powers and normalized powers throughout. This means that $\mathcal{R}_{A}(E)$ is normal.

Next let $\mathfrak{p} \subset A$ be a prime ideal of height at least two. We may assume that $A$ is local with maximal ideal $\mathfrak{m}=\mathfrak{p}$. To prove condition $\left(L_{2}\right)$ it suffices to show that height $\mathfrak{m} \mathcal{R}_{A}(E) \geq 2$. We will actually show that $\mathfrak{m} \mathcal{R}_{A}(E)$ has grade at least two. 
Since $A$ is normal of dimension at least two, its maximal ideal $\mathfrak{m}$ contains a regular sequence $a, b$ of length two. This sequence is regular on each of the $A$ modules $E^{i}$ because these modules are reflexive. Therefore $a, b$ form a regular sequence on the ring $\mathcal{R}_{A}(E)$. Obviously $a, b$ are contained in $\mathfrak{m} \mathcal{R}_{A}(E)$.

In the special case of projective dimension at most one, we can improve the above result. For this, we will rely on a basic property of integrally closed modules. Let $E$ be an integrally closed submodule of a free module $A^{r}$ over a normal domain $A$. The associated primes of $A^{r} / E$ are particularly restricted when $E$ is a module of finite projective dimension. This phenomenon was described in the case of ideals by Burch ([5, Corollary 3 on p. 947]). More recently, using a different approach, the question was taken up by Goto and Hayasaka ([7, 1.1 and 2.3]) in the case of ideals and by Hong $([12,4.8])$ in the case of modules. In the latter approaches the condition known as $\mathfrak{m}$-fullness was used. Let $(A, \mathfrak{m})$ be a Noetherian local ring with infinite residue field and let $E \subset A^{r}$ be a submodule of a free module. One says that $E$ is $\mathfrak{m}$-full if there exists an element $x \in \mathfrak{m}$ such that

$$
\mathfrak{m} E:_{A^{r}} x=E .
$$

The valuative criterion for integral dependence shows that an integrally closed module $E \subset A^{r}$ is $\mathfrak{m}$-full, provided the residue field of the normal local $\operatorname{ring} A$ is infinite.

We will make use of the following two results which are restated here for the reader's convenience. Most properties of the notion of $\mathfrak{m}$-fullness arise from the first of these results (see [29, 8.101] for details), including the second one.

Proposition 3.5. Let $(A, \mathfrak{m})$ be a Noetherian local ring and $E$ a submodule of $A^{r}$ such that $\mathfrak{m} E: A^{r} x=E$ for some $x \in \mathfrak{m}$. Then

$$
E / x E \simeq\left(E: A^{r} \mathfrak{m}\right) / E \oplus\left(E+x A^{r}\right) / x A^{r} \simeq\left(E: A^{r} \mathfrak{m}\right) / E \oplus E / x\left(E: A^{r} \mathfrak{m}\right) .
$$

Theorem 3.6. Let $(A, \mathfrak{m})$ be a Noetherian local ring and $E$ a submodule of $A^{r}$ such that $\mathfrak{m} E: A^{r} x=E$ for some regular element $x \in \mathfrak{m}$. Suppose that $\operatorname{projdim}(E)<\infty$ and $\mathfrak{m} \in \operatorname{Ass}\left(A^{r} / E\right)$. Then $A$ is regular.

In general, the normality of the Rees algebra $\mathcal{R}_{A}(E)$ of a finite module $E$ over a normal domain $A$ fails to imply that $E$ satisfies $\left(L_{2}\right)$ or $\left(F_{2}\right)$. This may be the case even if $E$ has projective dimension one. For an easy example take $E$ to be the homogeneous maximal ideal of a polynomial ring in two variables over a field. Surprisingly, the implication does hold for modules whose non-free locus is contained in the singular locus of the ring, as is stated in the next theorem.

Theorem 3.7. Let $A$ be a normal Cohen-Macaulay domain and let $E$ be a finite A-module of rank $r$ such that:

(i) $E$ has a projective resolution $0 \rightarrow P_{1} \longrightarrow P_{0} \longrightarrow E \rightarrow 0$;

(ii) $E_{\mathfrak{p}}$ is $A_{\mathfrak{p}}$-free for every prime $\mathfrak{p} \in \operatorname{Spec}(A)$ such that $A_{\mathfrak{p}}$ is regular. The following are equivalent:

(1) $\mathcal{R}_{A}(E)$ is normal;

(2) $\overline{E^{i}}=E^{i}$ in the range $1 \leq i \leq \operatorname{rank} P_{0}-r$;

(3) E satisfies condition $\left(F_{2}\right)$;

(4) $\mathcal{R}_{A}(E)=\mathcal{B}_{A}(E)$. 
Proof. (1) $\Rightarrow(2)$ : This is obvious.

$(2) \Rightarrow(3):$ Let $\mathfrak{p} \in \operatorname{Spec}(A)$ be a prime minimal with the property that $\left(F_{2}\right)$ fails. Localizing at $\mathfrak{p}$, we may assume that $A$ is a local ring of dimension $d \geq 2$, $\mu(E) \geq d+r-1$, and $E$ satisfies $\left(F_{2}\right)$ locally on the punctured spectrum of $A$. We may also suppose that the residue field of $A$ is infinite. Consider a minimal free resolution

$$
0 \rightarrow A^{s} \longrightarrow A^{n} \longrightarrow E \rightarrow 0 .
$$

By assumption $n \geq d+r-1$, hence $s \geq d-1$. Since $\left(F_{0}\right)$ holds on the punctured spectrum, it follows that the Weyman complex is a minimal free resolution of the symmetric power $\mathcal{S}_{d-1}(E)$ (see [31, Theorem 1(b)]). This complex has length $d-1$ because $s \geq d-1$. Moreover, $E$ satisfies $\left(F_{1}\right)$ on the punctured spectrum, therefore $\mathcal{S}_{d-1}(E)$ is torsionfree. Thus $E^{d-1} \simeq \mathcal{S}_{d-1}(E)$ has projective dimension $d-1$. In particular, $\mathfrak{m} \in \operatorname{Ass}\left(\mathcal{S}_{d-1}\left(A^{r}\right) / E^{d-1}\right)$ for any embedding $E^{d-1} \subset \mathcal{S}_{d-1}\left(A^{r}\right)$ as in (3.1). Since $d-1 \leq s \leq \operatorname{rank} P_{0}-r$, we can apply the main assumption to conclude that $E^{d-1}$ is integrally closed. Therefore $A$ is regular by Theorem 3.6. This is a contradiction vis-à-vis (ii) since $E$ is not free.

$(3) \Rightarrow(4)$ : This is well known for modules of projective dimension at most one over Cohen-Macaulay rings (see, e.g., [1, Proposition 4]).

$(4) \Rightarrow(1)$ : This follows from Proposition 3.4.

Theorem 3.7 applies naturally to the module $E=\Omega_{A / k}$ via the intervention of the Jacobian criterion, which says that the non-free locus of the module of differentials coincides with the singular locus of a reduced ring $A$ essentially of finite type over a perfect field $k$. If in addition $A$ is locally a complete intersection, then $E=\Omega_{A / k}$ also has projective dimension at most one.

Corollary 3.8. Let $R$ be a regular ring essentially of finite type over a perfect field, let $I$ be an $R$-ideal of height $g$ that is locally a complete intersection, and assume that $A=R / I$ is a normal domain. The following are equivalent:

(1) $\mathbb{R}_{A / k}$ is normal;

(2) $\overline{\Omega_{A / k}^{i}}=\Omega_{A / k}^{i}$ in the range $1 \leq i \leq g$;

(3) $\operatorname{edim} A_{\mathfrak{p}} \leq 2 \operatorname{dim} A_{\mathfrak{p}}-2$ for every non-regular prime $\mathfrak{p} \in \operatorname{Spec}(A)$;

(4) $\mathbb{R}_{A / k}=\mathbb{B}_{A / k}$.

Proof. Note that condition (3) translates into $\Omega_{A / k}$ satisfying $\left(F_{2}\right)$ according to the preliminaries of Section 2. The rest follows immediately from Theorem 3.7 .

The next result treats the case of rings that are not necessarily complete intersections. It is reminiscent of Theorem 2.10. We show that if a single symmetric power of $\Omega_{A / k}$ is torsionfree and integrally closed but not reflexive, then the defining ideal of $A$ must contain "quadrics".

Proposition 3.9. Let $R$ be a regular ring essentially of finite type over a perfect field $k$ and let $I$ be an ideal such that $A=R / I$ is a normal domain. If, for some $i \geq 1, \mathcal{S}_{i}\left(\Omega_{A / k}\right) \simeq \Omega_{A / k}^{i}$ and $\Omega_{A / k}^{i}$ is integrally closed, then $I \not \subset \mathfrak{p}^{(3)}$ for every $\mathfrak{p} \in \operatorname{Ass}_{R}\left(\left(\Omega_{A / k}^{i}\right)^{* *} / \Omega_{A / k}^{i}\right)$.

Proof. We replace $R$ by $R_{\mathfrak{p}}$ to assume that $(R, \mathfrak{n})$ and $(A, \mathfrak{m})$ are local. We may also suppose that the residue field is infinite. Notice that $\mathfrak{m} \in \operatorname{Ass}_{A}\left(\left(\Omega_{A / k}^{i}\right)^{* *} / \Omega_{A / k}^{i}\right)$; in 
particular, $\operatorname{dim} A \geq 2$. Since $\mathcal{S}_{i}\left(\Omega_{A / k}\right) \simeq \Omega_{A / k}^{i}$, the presentation

$$
I / I^{2} \longrightarrow A^{n}=A \otimes_{R} \Omega_{R / k} \longrightarrow \Omega_{A / k} \rightarrow 0
$$

induces an exact sequence

$$
I / I^{2} \otimes_{A} S_{i-1}\left(A^{n}\right) \longrightarrow S_{i}\left(A^{n}\right) \longrightarrow \Omega_{A / k}^{i} \rightarrow 0 .
$$

Embed $\Omega_{A / k}^{i} \subset\left(\Omega_{A / k}^{i}\right)^{* *} \subset A^{t}$. Since $\Omega_{A / k}^{i}$ is assumed to be integrally closed, it is $\mathfrak{m}$-full. Thus there is an element $x \in \mathfrak{m}$ satisfying the hypothesis of Proposition 3.5 with $E=\Omega_{A / k}^{i}$. Clearly $\mathfrak{m} \in \operatorname{Ass}_{A}\left(A^{t} / \Omega_{A / k}^{i}\right)$, hence $\Omega_{A / k}^{i}:_{A^{t}} \mathfrak{m} \neq \Omega_{A / k}^{i}$. Therefore the proposition implies that $\Omega_{A / k}^{i} / x \Omega_{A / k}^{i}$ has $A / \mathfrak{m}$ as a direct summand over $A /(x)$. On the other hand, tensoring (3.2) with $A /(x)$, we see that the syzygies of $\Omega_{A / k}^{i} / x \Omega_{A / k}^{i}$ have coefficients in the $A /(x)$-ideal generated by the entries of the Jacobian matrix of the generators of $I$. As $\mathfrak{m} /(x) \neq 0$, we cannot have $I \subset \mathfrak{n}^{3}$.

\section{StruCture Results in LOW CODIMENSION}

4.1. Normality revisited. We now focus on the behavior of $\mathbb{R}_{A / k}$ in low dimension or low embedding codimension. A highlight is the closer relationship between the normality of $\mathbb{R}_{A / k}$ and the local structure of $A$ than is typical of more general Rees algebras.

When $A$ is a Noetherian local $\operatorname{ring}$, we write ecodim $A:=\operatorname{edim} A-\operatorname{dim} A$.

Let $A$ be a local Cohen-Macaulay ring essentially of finite type over a perfect field $k$ and assume ecodim $A \leq 2$. Write $A \simeq R / I$, where $R$ is a regular local ring essentially of finite type over $k$ and $I$ an ideal of height $\leq 2$. In this case the first Koszul homology module $H_{1}(I)$ on a generating set of $I$ is Cohen-Macaulay (see 2, 2.1(a)]). Thus, if $I$ is generically a complete intersection, then the natural complex

$$
0 \rightarrow H_{1}(I) \longrightarrow A^{m} \longrightarrow I / I^{2} \rightarrow 0
$$

is exact. One always has a complex of graded modules over the polynomial ring $B:=\mathcal{S}_{A}\left(\Omega_{R / k} \otimes_{R} A\right) \simeq \mathcal{S}_{A}\left(A^{n}\right)$,

$$
0 \rightarrow \operatorname{Tor}_{2}^{R}(A, A) \otimes_{A} B(-2) \longrightarrow \operatorname{Tor}_{1}^{R}(A, A) \otimes_{A} B(-1) \longrightarrow B \longrightarrow \mathbb{S}_{A / k} \rightarrow 0,
$$

which is nothing but the $\mathcal{M}$-complex of the diagonal ideal $\mathbb{D}_{A / k} \subset A \otimes_{k} A$ (see 22, Section 2.2] for details). If height $I \leq 1$, then the module $\operatorname{Tor}_{2}^{R}(A, A)$ vanishes, whereas for height $I=2$ it is isomorphic to $\left(\omega_{A}\right)^{*}$, the $A$-dual of the canonical module of $A$. The complex (4.2) can be used to derive properties of $\mathbb{S}_{A / k}$ and of $\mathbb{R}_{A / k}$ :

Proposition 4.1. Let $A$ be a reduced local Cohen-Macaulay ring essentially of finite type over a perfect field $k$. Assume that ecodim $A \leq 2$ and that $\operatorname{edim} A_{\mathfrak{p}} \leq$ $2 \operatorname{dim} A_{\mathfrak{p}}$ for every prime $\mathfrak{p} \in \operatorname{Spec}(A)$.

(a) The sequence (4.2) is exact, and $\mathbb{S}_{A / k}$ is Cohen-Macaulay.

(b) $\Omega_{A / k}$ is of linear type if and only if $\operatorname{edim} A_{\mathfrak{p}} \leq 2 \operatorname{dim} A_{\mathfrak{p}}-1$ for every non-minimal prime $\mathfrak{p} \in \operatorname{Spec}(A)$.

(c) In case $A$ is normal, $\Omega_{A / k}$ is of linear type and normal if and only if $\mathbb{S}_{A / k}=\mathbb{B}_{A / k}$ if and only if edim $A_{\mathfrak{p}} \leq 2 \operatorname{dim} A_{\mathfrak{p}}-2$ for every non-regular prime $\mathfrak{p} \in \operatorname{Spec}(A)$. 
Proof. Write $A=R / I$ as above, with height $I=2$.

(a) Notice that

$$
\left(\omega_{A}\right)^{*} \simeq \operatorname{Hom}_{A}\left(\omega_{A} \otimes_{A} \omega_{A}, \omega_{A}\right) \simeq \operatorname{Hom}_{A}\left(S_{2}\left(\omega_{A}\right), \omega_{A}\right) .
$$

By [30, 1.3], $S_{2}\left(\omega_{A}\right)$ is a maximal Cohen-Macaulay $A$-module, hence $\operatorname{Tor}_{2}^{R}(A, A)$ is zero or maximal Cohen-Macaulay. Moreover, (4.1) gives depth $I / I^{2} \geq \operatorname{dim} A-1$. Since these depth conditions also hold locally at every prime and since $\Omega_{A / k}$ satisfies condition $\left(F_{0}\right)$, the Acyclicity Lemma implies that (4.2) is exact. It also follows that $\operatorname{depth} \mathbb{S}_{A / k} \geq \operatorname{dim} A+n-2$. On the other hand, $\operatorname{dim} \mathbb{S}_{A / k}=\operatorname{dim} A+n-2$ again because $\Omega_{A / k}$ satisfies $\left(F_{0}\right)$ (see [24, 2.2]). Therefore $\mathbb{S}_{A / k}$ is Cohen-Macaulay.

(b) From part (a) we have that $\mathbb{S}_{A / k}$ is unmixed. In this case, the module $\Omega_{A / k}$ is of linear type if and only if it satisfies $\left(F_{1}\right)$ (see [24, 3.3 and the first remark on p. 346]). Alternatively, one could use the exact sequence (4.2).

(c) Assume that $\Omega_{A / k}$ is of linear type and normal. We show the equality $\mathbb{S}_{A / k}=$ $\mathbb{B}_{A / k}$. For any non-regular prime $\mathfrak{p} \in \operatorname{Spec}(A)$ write $A_{\mathfrak{p}} \simeq R / I$, where $(R, \mathfrak{n})$ is a regular local ring essentially of finite type over $k$ and $I \subset \mathfrak{n}^{2}$ is an ideal of height $g \leq 2$. By Theorem 2.10, it suffices to show that $\mu\left(I+\mathfrak{n}^{3} / \mathfrak{n}^{3}\right) \leq 2 g-1$. This is clear if $g=1$ or if $g=2$ and $\mu(I) \leq 3$. Thus we may assume that $g=2$ and $\mu(I) \geq 4$. But in this situation the Hilbert-Burch theorem gives $I \subset \mathfrak{n}^{3}$.

If $\mathbb{S}_{A / k}=\mathbb{B}_{A / k}$, then $\Omega_{A / k}$ is of linear type and, in particular, free locally in codimension one. Hence the conditions $\left(F_{2}\right)$ and $\left(L_{2}\right)$ are equivalent. Thus Proposition 3.4 shows that $\Omega_{A / k}$ satisfies $\left(F_{2}\right)$.

Finally, if $\Omega_{A / k}$ has the $\left(F_{2}\right)$ property, then it is of linear type by part (b). Moreover, part (a) implies that $\mathbb{S}_{A / k}$ is Cohen-Macaulay. Thus $\mathbb{S}_{A / k}=\mathbb{R}_{A / k}$ satisfies condition (2) of Proposition 3.4, hence the proposition implies that $\Omega_{A / k}$ is normal.

We now treat the case where $A$ is a local Gorenstein algebra of embedding codimension 3. Write $A \simeq R / I$, where $R$ is a regular local ring essentially of finite type over a perfect field $k$ and $I$ is an ideal of height 3. By [8, 2.8], $I / I^{2}$ is a maximal Cohen-Macaulay $A$-module and therefore $\left(I / I^{2}\right)^{* *} \simeq I / I^{2}$. Thus, starting from a presentation of $\Omega_{A / k}$, we obtain the $\mathcal{Z}$-complex

$$
\begin{aligned}
& 0 \rightarrow\left(\wedge^{\wedge} I / I^{2}\right)^{* *} \otimes_{A} B(-3) \longrightarrow\left({ }^{2} I / I^{2}\right)^{* *} \otimes_{A} B(-2) \longrightarrow\left(I / I^{2}\right) \otimes_{A} B(-1) \\
& \longrightarrow B \longrightarrow \mathbb{S}_{A / k} \rightarrow 0,
\end{aligned}
$$

where $B$ is the polynomial ring $\mathcal{S}_{A}\left(\Omega_{R / k} \otimes_{R} A\right)$.

Proposition 4.2. Let $A$ be a reduced local Gorenstein ring essentially of finite type over a perfect field $k$. Assume that ecodim $A \leq 3$ and that $\operatorname{edim} A_{\mathfrak{p}} \leq 2 \operatorname{dim} A_{\mathfrak{p}}$ for every prime $\mathfrak{p} \in \operatorname{Spec}(A)$.

(a) The sequence (4.3) is exact, and $\mathbb{S}_{A / k}$ is Gorenstein.

(b) $\Omega_{A / k}$ is of linear type if and only if edim $A_{\mathfrak{p}} \leq 2 \operatorname{dim} A_{\mathfrak{p}}-1$ for every non-minimal prime $\mathfrak{p} \in \operatorname{Spec}(A)$.

(c) In case $A$ is normal, $\Omega_{A / k}$ is of linear type and normal if and only if $\mathbb{S}_{A / k}=\mathbb{B}_{A / k}$ if and only if edim $A_{\mathfrak{p}} \leq 2 \operatorname{dim} A_{\mathfrak{p}}-2$ for every non-regular prime $\mathfrak{p} \in \operatorname{Spec}(A)$.

Proof. Since edim $A_{\mathfrak{p}} \leq 2 \operatorname{dim} A_{\mathfrak{p}}$ for every prime $\mathfrak{p} \in \operatorname{Spec}(A)$ and $A$ is Gorenstein, it follows that $A$ is a complete intersection locally in codimension two. We note 
that $\left(\bigwedge^{3} I / I^{2}\right)^{* *} \simeq\left(\omega_{A}\right)^{*} \simeq A$ and that the pairing

$$
\left(\stackrel{2}{\wedge} I / I^{2}\right) \times I / I^{2} \longrightarrow \stackrel{3}{\wedge} I / I^{2} \longrightarrow A
$$

identifies $\left(\bigwedge^{2} I / I^{2}\right)^{* *}$ with $\left(I / I^{2}\right)^{*}$. As $I / I^{2}$ is a maximal Cohen-Macaulay $A$ module, we conclude that the three leftmost modules in (4.3) are maximal CohenMacaulay $B$-modules. Therefore (4.3) is exact and, since it is a self-dual complex of $B$-modules, $\mathbb{S}_{A / k}$ is Gorenstein. Now the argument proceeds as in the proof of Proposition 4.1. To show (c) one replaces the Hilbert-Burch theorem used there by the Buchsbaum-Eisenbud structure theorem.

One ought to observe that parts (a) and (b) of Propositions 4.1 and 4.2 were obtained in [22, 3.4 and 3.2] by passing through the tangent star algebra $\mathbb{T}_{A / k}$. Since the present approach is self-contained and perhaps more direct, we have chosen to keep it for completeness.

The previous results motivate the following question:

Question 4.3. Let $A$ be a normal Cohen-Macaulay domain essentially of finite type over a perfect field $k$. If $\Omega_{A / k}$ is of linear type and normal, does it follow that $\operatorname{edim} A_{\mathfrak{p}} \leq 2 \operatorname{dim} A_{\mathfrak{p}}-2$ for every non-regular prime $\mathfrak{p} \in \operatorname{Spec}(A)$ (or, equivalently, is $\left.\mathbb{S}_{A / k}=\mathbb{B}_{A / k}\right)$ ?

Our aim for most of the remainder of the section is a refined version of Proposition 4.1(c) that does not require the linear type assumption on the module of differentials; see Theorem 4.8 below. For this we need several auxiliary results that may be of independent interest. Recall that if $A$ is a Noetherian ring and $U \subset E$ are finite modules having a rank, then $U$ is said to be a reduction of $E$ if the induced inclusion $\mathcal{R}(U) \subset \mathcal{R}(E)$ is an integral ring extension (see, e.g., [23, p. 613]).

Proposition 4.4. Let $A$ be a Noetherian ring and let $\varphi: A^{g+1} \longrightarrow A^{g}$ be a homomorphism such that $\operatorname{im}(\varphi)$ has rank $g$ and $\operatorname{Ext}_{A}^{1}(\operatorname{im}(\varphi), A)=0$. Then $I_{g}(\varphi) \simeq$ $(\operatorname{ker}(\varphi))^{*}$. In particular, if $A$ satisfies $\left(S_{2}\right)$, it follows that $I_{g}(\varphi)$ is either the unit ideal or an unmixed ideal of height one. If in addition $A$ is Gorenstein locally in codimension one, then $\operatorname{im}(\varphi)$ is a reduction of $(\operatorname{im}(\varphi))^{* *}$.

Proof. There is an exact sequence

$$
A^{n} \stackrel{\psi}{\longrightarrow} A^{g+1} \stackrel{\varphi}{\longrightarrow} A^{g},
$$

so that the entries of the first column of $\psi$ are the signed maximal minors of $\varphi$; hence they generate $I_{g}(\varphi)$, an ideal of positive grade. Set $K:=\operatorname{ker}(\varphi)$ and $L:=\operatorname{im}(\varphi)$. From the assumption we obtain a short exact sequence

$$
0 \rightarrow L^{*} \longrightarrow A^{g+1^{*}} \longrightarrow K^{*} \rightarrow 0
$$

which shows that $K^{*} \simeq \operatorname{im}\left(\psi^{*}\right)$. Next we project $A^{n *}$ onto the free module generated by the first basis element, thus getting a commutative diagram

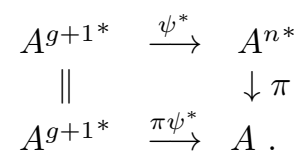

Notice that $\operatorname{im}\left(\pi \psi^{*}\right)=I_{g}(\varphi)$. The projection $\pi$ induces a surjection $\operatorname{im}\left(\psi^{*}\right) \rightarrow$ $\operatorname{im}\left(\pi \psi^{*}\right)=I_{g}(\varphi)$, which is necessarily an isomorphism since the two modules are torsionfree of rank one. 
Moreover, if $A$ satisfies $\left(S_{2}\right)$, then $I_{g}(\varphi)$ satisfies $\left(S_{2}\right)$; hence it is the unit ideal or an unmixed ideal of height one. The last assertion is a property of the integral closure of modules with divisorial determinantal ideal (see [12, the proof of 2.5]).

Corollary 4.5. Let $R$ be a regular local ring and let $A=R / I$ be an almost complete intersection which is a complete intersection locally in codimension one and satisfies $\left(S_{2}\right)$. If $A$ is non-obstructed (i.e., $\left.T^{2}(A / R, A)=\operatorname{Ext}_{A}^{1}\left(I / I^{2}, A\right)=0\right)$, then $I / I^{2}$ is a reduction of $\left(I / I^{2}\right)^{* *}$.

Proof. Write $g:=$ height $I$. First notice that $A$ is equidimensional because it is catenary and satisfies $\left(S_{2}\right)$. Therefore $I / I^{2}$ has rank $g$ as an $A$-module. Since $I$ is generated by $g+1$ elements, the first Koszul homology $H_{1}(I)$ of these elements is the canonical module of $A$, hence satisfies $\left(S_{2}\right)$. Therefore the sequence

$$
0 \rightarrow H_{1}(I) \longrightarrow A^{g+1} \longrightarrow I / I^{2} \rightarrow 0
$$

is exact and $I / I^{2}$ is a torsionfree $A$-module. Embedding $I / I^{2}$ into $A^{g}$ and applying Proposition 4.4 with $\operatorname{im}(\varphi)=I / I^{2}$, we deduce the result.

Next is a curious result in embedding codimension 2 that, like Theorem 2.6, can be regarded as an analogue of Berger's conjecture.

As in previous instances, $\tau_{A}(E)$ will denote the $A$-torsion of an $A$-module $E$.

Let $A=R / I$ be a normal Cohen-Macaulay ring, where $R$ is a regular local ring essentially of finite type over a perfect field $k$ and height $I \leq 2$. The exact sequence (4.1) and the Cohen-Macaulayness of $H_{1}(I)$ imply that $I / I^{2}$ is torsionfree. Thus the natural complex

$$
0 \rightarrow I / I^{2} \longrightarrow \Omega_{R / k} \otimes_{R} A \simeq A^{n} \longrightarrow \Omega_{A / k} \rightarrow 0
$$

is exact as well.

Theorem 4.6. Let $A$ be a normal local Cohen-Macaulay ring essentially of finite type over a perfect field $k$. Assume the following conditions:

(i) $\operatorname{ecodim} A \leq 2$;

(ii) $A$ is an almost complete intersection locally in codimension 2.

If $\Omega_{A / k} / \tau_{A}\left(\Omega_{A / k}\right)$ is a Cohen-Macaulay module, then $A$ is regular.

Proof. Set $\Omega:=\Omega_{A / k}$ and $\omega:=\omega_{A}$. Write $A \simeq R / I$, where $R$ is a regular local ring essentially of finite type over $k$ and $I$ a perfect ideal of height 2 . It suffices to prove that $I$ is a complete intersection, for in this case (4.4) shows that $\Omega$ is torsionfree of projective dimension at most one. Thus $\Omega=\Omega / \tau_{A}(\Omega)$ is a maximal Cohen-Macaulay module of finite projective dimension, hence necessarily free.

We first consider the case $\operatorname{dim} A=2$. Supposing $I$ is not a complete intersection we have that this ideal is minimally generated by 3 elements. Consider a minimal presentation

$$
0 \rightarrow R^{2} \stackrel{\varphi}{\longrightarrow} R^{3} \longrightarrow I \rightarrow 0 \text {. }
$$

From the exact sequences (4.1) and (4.4) we derive an exact sequence

$$
0 \rightarrow \omega \simeq H_{1}(I) \longrightarrow A^{3} \stackrel{\Theta}{\longrightarrow} A^{n} \longrightarrow \Omega \rightarrow 0,
$$

with $\Theta$ the transposed Jacobian matrix of the 3 generators of $I$. Moreover, since $\Omega / \tau_{A}(\Omega)$ is Cohen-Macaulay and $\tau_{A}(\Omega)$ has grade at least 2, one concludes that 
$\operatorname{Ext}_{A}^{1}(\Omega, \omega)=0$. Thus, applying $-^{\vee}=\operatorname{Hom}_{A}(-, \omega)$ to (4.6), one can see that there is an exact sequence

$$
\omega^{n} \stackrel{\Theta^{\vee}}{\longrightarrow} \omega^{3} \longrightarrow \omega^{\vee} \simeq A \longrightarrow A / I_{1}(\varphi) A \rightarrow 0 .
$$

Hence we obtain the exact sequence

$$
\omega^{n} \stackrel{\Theta^{\vee}}{\longrightarrow} \omega^{3} \longrightarrow I_{1}(\varphi) / I \rightarrow 0 .
$$

By (4.5) and the Hilbert-Burch theorem, one has $I \subset I_{1}(\varphi)^{2}$. In particular, $I_{1}(\Theta) \subset I_{1}(\varphi)$. Thus tensoring (4.7) with $R / I_{1}(\varphi)$ gives

$$
\left(\omega / I_{1}(\varphi) \omega\right)^{\oplus 3} \simeq\left(I_{1}(\varphi) / I\right) \otimes_{R} R / I_{1}(\varphi) \simeq I_{1}(\varphi) / I_{1}(\varphi)^{2} .
$$

On the other hand, dualizing (4.5) into $R$ and tensoring with $R / I_{1}(\varphi)$ yields

$$
\omega / I_{1}(\varphi) \omega \simeq\left(R / I_{1}(\varphi)\right)^{\oplus 2} .
$$

It follows that

$$
I_{1}(\varphi) / I_{1}(\varphi)^{2} \simeq\left(\omega / I_{1}(\varphi) \omega\right)^{\oplus 3} \simeq\left(R / I_{1}(\varphi)\right)^{\oplus 6} .
$$

Since $R$ is regular, [27, 1.1] implies that $I_{1}(\varphi)$ is generated by a regular sequence of length 6 . But this is impossible in $R$, a ring of dimension four.

We now consider the case of arbitrary dimension. By the above, $A$ is regular locally in codimension 2. Therefore (4.6) implies that $\Omega$ is torsionfree, hence CohenMacaulay by assumption. But then also $I / I^{2}$ is Cohen-Macaulay by (4.4), which forces $I$ to be a complete intersection (see [8, 2.4]).

The next proposition leads into our main result about the normality of the module of differentials in the case of embedding codimension two.

Proposition 4.7. Let $A$ be a normal local ring essentially of finite type over a perfect field $k$. Assume the following conditions:

(i) $\operatorname{dim} A=\operatorname{ecodim} A=2$;

(ii) $A$ is either a complete intersection or else an almost complete intersection defined by an ideal of order $\geq 3$.

Then $\Omega_{A / k}$ is not integrally closed.

Proof. Write $A \simeq R / I$, where $(R, \mathfrak{n})$ is a regular local ring essentially of finite type over $k$ and $I$ a perfect ideal of height 2. We may assume that $R$ has infinite residue field. Let $\mathfrak{m}$ denote the maximal ideal of $A$. Set $\Omega:=\Omega_{A / k}$ and $\Omega^{1}:=\Omega / \tau_{A}(\Omega)$.

There is an exact sequence

$$
0 \rightarrow\left(I / I^{2}\right)^{* *} \longrightarrow A^{n} \longrightarrow \Omega^{1} \rightarrow 0 \text {. }
$$

By Theorem [4.6. $\Omega^{1}$ is not Cohen-Macaulay. Thus $\mathfrak{m} \in \operatorname{Ass}_{A}\left(A^{r} / \Omega^{1}\right)$ for any embedding $\Omega^{1} \subset A^{r}$. Suppose that $\Omega$ is integrally closed, which means that $\Omega^{1}$ is integrally closed. In particular, $\Omega^{1}$ is $\mathfrak{m}$-full. If $A$ is a complete intersection, then $\Omega^{1}$ has finite projective dimension by (4.8), which leads to a contradiction according to Theorem 3.6. Thus we may assume that $A$ is an almost complete intersection and that $I \subset \mathfrak{n}^{3}$. By Proposition 3.5, there exists an $x \in \mathfrak{m}$ such that $A / \mathfrak{m}$ is a direct summand of $\Omega^{1} / x \Omega^{1}$. Write $\left(A^{\prime}, \mathfrak{m}^{\prime}\right)$ for the local ring $A /(x)$ and let $\left\{e_{1}, \ldots, e_{n}\right\}$ be the canonical basis of $A^{\prime n}$. Tensoring (4.8) with $A^{\prime}$ we obtain a presentation

$$
0 \rightarrow M \longrightarrow A^{\prime n} \longrightarrow \Omega^{1} / x \Omega^{1} \rightarrow 0,
$$


where we may assume that $M=\mathfrak{m}^{\prime} e_{1} \oplus N$ with $N \subset \oplus_{i=2}^{n} A^{\prime} e_{i}$. Hence the image of $M$ under the projection $A^{\prime n} \rightarrow A^{\prime} e_{1} \simeq A^{\prime}$ is $\mathfrak{m}^{\prime}$.

On the other hand, as $A$ is a Cohen-Macaulay ring of embedding codimension 2, it is non-obstructed (see [9, 3.2(a)]). Therefore by Corollary 4.5, $I / I^{2}$ is a reduction of $\left(I / I^{2}\right)^{* *}$. Since $I \subset \mathfrak{n}^{3}$, it follows that $I / I^{2} \subset \mathfrak{m}^{2} A^{n}$. Hence, projecting onto $A^{\prime} e_{1} \simeq A^{\prime}$, we see that $\left(\mathfrak{m}^{\prime}\right)^{2}$ is a reduction of $\mathfrak{m}^{\prime}$. This is impossible because $\operatorname{dim} A^{\prime}>0$.

Theorem 4.8. Let $A$ be a normal local Cohen-Macaulay ring essentially of finite type over a perfect field $k$. Assume the following conditions:

(i) $\operatorname{ecodim} A \leq 2$;

(ii) Locally in codimension 2, the ring $A$ is either a complete intersection or else an almost complete intersection defined by an ideal of order $\geq 3$.

Then the following are equivalent:

(1) $\Omega_{A / k}$ is normal;

(2) $\operatorname{edim} A_{\mathfrak{p}} \leq 2 \operatorname{dim} A_{\mathfrak{p}}-2$ for every non-regular prime $\mathfrak{p} \in \operatorname{Spec}(A)$.

Moreover, if the equivalent conditions (1) or (2) hold, then $\Omega_{A / k}$ is of linear type, $\mathbb{R}_{A / k}$ is Cohen-Macaulay, and $\mathbb{R}_{A / k}=\mathbb{B}_{A / k}$.

Proof. (1) $\Rightarrow(2)$ : Proposition 4.7 implies that $\Omega_{A / k}$ satisfies $\left(F_{1}\right)$. Hence by Proposition 4.1(b), $\Omega_{A / k}$ is of linear type, and then according to Proposition 4.1(c), it satisfies $\left(F_{2}\right)$.

$(2) \Rightarrow(1)$ : This follows from Proposition 4.1(c).

The remaining assertions follow from the same proposition.

4.2. Relation to Calabi-Yau varieties. In this last part we explain to what extent the present results relate to Calabi-Yau varieties.

Let $X \subset \mathbb{P}_{\mathbb{C}}^{n-1}$ be an arithmetically normal projective variety and let $A$ stand for its homogeneous coordinate ring. We say that $X$ is of Calabi-Yau type if there exists a homogeneous isomorphism $\omega_{A} \simeq A$. The notion of Calabi-Yau variety would also require that $X$ be smooth and $H^{1}\left(X, \mathcal{O}_{X}\right)=0$. If $X$ is of Calabi-Yau type, it often turns out that $\operatorname{Proj}\left(\mathbb{R}_{A / \mathbb{C}}\right) \subset \mathbb{P}_{\mathbb{C}}^{2 n-1}$ has the same property. Here and in what follows we use the natural embeddings $\operatorname{Proj}\left(\mathbb{R}_{A / \mathbb{C}}\right) \subset \operatorname{Proj}\left(\mathbb{S}_{A / \mathbb{C}}\right) \subset \operatorname{Proj}\left(\mathbb{S}_{R / \mathbb{C}}\right)=\mathbb{P}_{\mathbb{C}}^{2 n-1}$, where $R=\mathbb{C}\left[X_{1}, \ldots, X_{n}\right]$ and $\mathbb{S}_{R / \mathbb{C}}$ is a polynomial ring in $2 n$ variables over $\mathbb{C}$ that is regarded as standard graded.

We first look into the case of complete intersections.

Proposition 4.9. Let $X \subset \mathbb{P}_{\mathbb{C}}^{n-1}$ be a non-degenerate smooth projective variety that is a complete intersection of $g \geq 1$ hypersurfaces of degrees $d_{1} \geq \cdots \geq d_{g}$, and let $A$ stand for its homogeneous coordinate ring. Assume that $X$ is of Calabi-Yau type and consider the subschemes $Y:=\operatorname{Proj}\left(\mathbb{R}_{A / \mathbb{C}}\right) \subset Z:=\operatorname{Proj}\left(\mathbb{S}_{A / \mathbb{C}}\right) \subset \mathbb{P}_{\mathbb{C}}^{2 n-1}$.

(a) The subscheme $Z$ is the complete intersection of $2 g$ hypersurfaces of degrees $d_{1}, \ldots, d_{g}, d_{1}, \ldots, d_{g}$ and $\omega_{\mathbb{S}_{A / \mathbb{C}}} \simeq \mathbb{S}_{A / \mathbb{C}}$ as graded modules.

(b) If $d_{1}=2$, then $Z$ is neither reduced nor irreducible, and $Y$ is not arithmetically Cohen-Macaulay.

(c) If $d_{1} \geq 3$, then $Y=Z$ is reduced and irreducible.

(d) The subscheme $Y$ is arithmetically normal if and only if $d_{1} \geq 4$ or $d_{2} \geq 3$ (formally setting $d_{2}=2$ when $g=1$ ). In this case $Y=Z$ is of Calabi-Yau type. 
Proof. Since $\omega_{A} \simeq A\left(-n+\sum_{i=1}^{g} d_{i}\right)$ and $X$ is of Calabi-Yau type, we have $n=$ $\sum_{i=1}^{g} d_{i}$. Since $X$ is non-degenerate, $d_{g} \geq 2$. Therefore $n \geq 2 g$, hence $\Omega_{A / \mathbb{C}}$ satisfies condition $\left(F_{0}\right)$. Since $\operatorname{projdim}\left(\Omega_{A / \mathbb{C}}\right) \leq 1$, this implies that $\mathbb{S}_{A / \mathbb{C}}$ is a complete intersection of dimension $2(n-g)$ (see [1, Proposition 4]). Moreover, $\mathbb{S}_{A / \mathbb{C}}$ is defined by $2 g$ forms of degrees $d_{1}, \ldots, d_{g}, d_{1}, \ldots, d_{g}$ in a standard graded polynomial ring in $2 n$ variables over $\mathbb{C}$. Therefore $\omega_{\mathbb{S}_{A / \mathbb{C}}} \simeq \mathbb{S}_{A / \mathbb{C}}\left(-2 n+2 \sum_{i=1}^{g} d_{i}\right)=\mathbb{S}_{A / \mathbb{C}}$ as graded modules. This shows (a).

To prove (b), note that the hypothesis forces the equality $n=2 g$ and thus $\Omega_{A / \mathbb{C}}$ does not satisfy condition $\left(F_{1}\right)$. It follows from [4, the proof of 2.2 ] that $Z$ is not irreducible, whereas Theorem 2.1 (or Remark 2.3) implies that $Z$ is not reduced either. Furthermore, $Y$ is not arithmetically Cohen-Macaulay according to Theorem 3.1 (or Remark 3.2).

As to (c), the assumption implies that $n \geq 2 g+1$; hence $\Omega_{A / \mathbb{C}}$ satisfies condition $\left(F_{1}\right)$. Again since projdim $\left(\Omega_{A / \mathbb{C}}\right) \leq 1$ we have $\mathbb{S}_{A / \mathbb{C}}=\mathbb{R}_{A / \mathbb{C}}$ (see [1, Proposition 4], [13, 1.1], 24, 3.4]). Finally, notice that $d_{1} \geq 4$ or $d_{2} \geq 3$ if and only if $n \geq 2 g+2$. Thus the equivalence in (d) follows from Corollary 3.8. Parts (a) and (c) then imply the rest.

Next is the non-complete intersection case.

Proposition 4.10. Let $X \subset \mathbb{P}_{\mathbb{C}}^{n-1}$ be a smooth arithmetically Cohen-Macaulay projective variety that is not a complete intersection, and let $A$ stand for its homogeneous coordinate ring. Assume that $\operatorname{dim} X \geq 2$, ecodim $X \leq 3$, and $X$ is of CalabiYau type. Consider the subschemes $Y:=\operatorname{Proj}\left(\mathbb{R}_{A / \mathbb{C}}\right) \subset Z:=\operatorname{Proj}\left(\mathbb{S}_{A / \mathbb{C}}\right) \subset \mathbb{P}_{\mathbb{C}}^{2 n-1}$.

(a) The subscheme $Z$ is arithmetically Gorenstein and $\omega_{\mathbb{S}_{A / \mathbb{C}}} \simeq \mathbb{S}_{A / \mathbb{C}}$ as graded modules.

(b) If $\operatorname{dim} X=2$, then $Z$ is neither reduced nor irreducible.

(c) If $\operatorname{dim} X \geq 3$, then $Y=Z$ is reduced and irreducible.

(d) The subscheme $Z$ is arithmetically normal if and only if $\operatorname{dim} X \geq 4$, in which case $Y=Z$ is of Calabi-Yau type.

Proof. Notice that $A \simeq R / I$, where $R=\mathbb{C}\left[X_{1}, \ldots, X_{n}\right]$ and $I \subset\left(X_{1}, \ldots, X_{n}\right)^{2}$ is a homogeneous Gorenstein ideal of height at most 3 , hence of height 3 because $X$ is not a complete intersection. Since $X$ is smooth and $n=\operatorname{height} I+\operatorname{dim} A \leq$ $2 \operatorname{dim} A$, the module $\Omega_{A / \mathbb{C}}$ satisfies $\left(F_{0}\right)$. Proposition 4.2 (a) then shows that $\mathbb{S}_{A / \mathbb{C}}$ is Gorenstein and the complex (4.3) is exact. In fact, as a complex of graded modules over the standard graded $\mathbb{C}$-algebra $B:=A\left[T_{1}, \ldots, T_{n}\right]$, this exact sequence now reads

$$
\begin{aligned}
0 \longrightarrow & \left(\wedge^{3} I / I^{2}\right)^{* *} \otimes_{A} B \longrightarrow\left(\wedge^{2} I / I^{2}\right)^{* *} \otimes_{A} B \longrightarrow\left(I / I^{2}\right) \otimes_{A} B \\
\longrightarrow & B \longrightarrow \mathbb{S}_{A / \mathbb{C}} \rightarrow 0,
\end{aligned}
$$

where $-^{*}=\operatorname{Hom}_{A}(-, A)=\operatorname{Hom}_{A}\left(-, \omega_{A}\right)$. One has $\left(\bigwedge^{3} I / I^{2}\right)^{* *} \simeq \operatorname{Tor}_{3}^{R}(A, A) \simeq$ $A(-n)$. Now the arguments in the proof of Proposition 4.2 (a) show that $\omega_{\mathbb{S}_{A / \mathbb{C}}} \simeq$ $\mathbb{S}_{A / \mathbb{C}}$ as graded modules, proving part (a).

As to (b), if $\operatorname{dim} X=2$, then $n=$ height $I+\operatorname{dim} A=6=2 \operatorname{dim} A$. In particular, $\Omega_{A / \mathbb{C}}$ does not satisfy $\left(F_{1}\right)$. Thus $Z$ is not irreducible according to 4 , the proof of 2.2 . On the other hand, by considering the Hilbert function of $A$ modulo a linear system of parameters, one sees that $\operatorname{dim}_{\mathbb{C}}[I]_{2} \leq 5=n-1$. Now an application of Theorem 2.1 (or Remark 2.3) yields that $Z$ is not reduced either, proving (b). 
Finally, part (c) follows from Proposition 4.2(b) and part (d) from Proposition 4.2 (c).

\section{ACKNOWLEDGEMENTS}

The first author thanks the Department of Mathematics at Purdue University for the hospitality during the preparation of part of this work. The second and third authors thank the Departamento de Matemática at the Universidade Federal de Pernambuco (Brazil) for the hospitality during various visits.

\section{REFERENCES}

1. L. Avramov, Complete intersections and symmetric algebras, J. Algebra 73 (1981), 248-263. MR 641643 (83e:13024)

2. L. Avramov and J. Herzog, The Koszul algebra of a codimension 2 embedding, Math. Z. 175 (1980), 249-260. MR602637 (82g:13011)

3. R. Berger, Differentialmoduln eindimensionaler lokaler Ringe, Math. Z. 81 (1963), 326-354. MR0152546 (27:2524)

4. J. Brennan, M. V. Pinto and W. V. Vasconcelos, The Jacobian module of a Lie algebra, Trans. Amer. Math. Soc. 321 (1990), 183-196. MR.958883 (90m:13023)

5. L. Burch, On ideals of finite homological dimension in local rings, Math. Proc. Cambridge Philos. Soc. 64 (1968), 941-948. MR0229634 (37:5208)

6. A. Corso, C. Polini and B. Ulrich, Core of projective dimension one modules, Manuscripta Math. 111 (2003), 427-433. MR2002819 (2004h:13006)

7. S. Goto and F. Hayasaka, Finite homological dimension and primes associated to integrally closed ideals, Proc. Amer. Math. Soc. 130 (2002), 3159-3164. MR.1912992 (2003g:13014)

8. J. Herzog, Ein Cohen-Macaulay Kriterium mit Anwendungen auf den Konormalenmodul und den Differentialmodul, Math. Z. 163 (1978), 149-162. MR512469 (80a:13025)

9. J. Herzog, Deformationen von Cohen-Macaulay Algebren, J. Reine Angew. Math. 318 (1980), 83-105. MR579384 (81m:13012)

10. J. Herzog, A. Simis and W. V. Vasconcelos, Koszul homology and blowing-up rings, in Commutative Algebra, Lecture Notes in Pure and Applied Math. 84, Marcel-Dekker, New York, 1983, 79-169. MR686942 (84k:13015)

11. J. Herzog, A. Simis and W. V. Vasconcelos, On the arithmetic and homology of algebras of linear type, Trans. Amer. Math. Soc. 283 (1984), 661-683. MR737891 (86a:13015)

12. J. Hong, S. Noh and W. V. Vasconcelos, Integrally closed modules and their divisors, Comm. Algebra 33 (2005), 4719-4733. MR2188337 (2007d:13011)

13. C. Huneke, On the symmetric algebra of a module, J. Algebra 69 (1981), 113-119. MR613861 (82d:13016)

14. C. Huneke, A. Simis and W. V. Vasconcelos, Reduced normal cones are domains, Contemp. Math. 88 (1989), 95-101. MR.999985 (90c:13010)

15. M. Johnson, Depth of symmetric algebras of certain ideals, Proc. Amer. Math. Soc. 129 (2001), 1581-1585. MR1814083 (2002b:13006)

16. J. Lipman, On the Jacobian ideal of the module of differentials, Proc. Amer. Math. Soc. 21 (1969), 422-426. MR0237511(38:5793)

17. P. Roberts, An infinitely generated symbolic blow-up in a power series ring and a new counterexample to Hilbert's Fourteenth Problem, J. Algebra 132 (1990), 461-473. MR1061491 (91j:13006)

18. G. Scheja and U. Storch, Über differentielle Abhängigkeit bei Idealen analytischer Algebren, Math. Z. 114 (1970), 101-112. MR0263808 (41:8408)

19. A. Simis, Remarkable Graded Algebras in Algebraic Geometry, XII ELAM, IMCA, Lima, Peru, 1999. MR2007333

20. A. Simis, Two differential themes in characteristic zero, Contemp. Math. 324 (2003), 195-204. MR.1986124

21. A. Simis, K. Smith and B. Ulrich, An algebraic proof of Zak's inequality for the dimension of the Gauss image, Math. Z. 241 (2002), 871-881. MR.1942243(2004a:13002)

22. A. Simis, B. Ulrich and W. V. Vasconcelos, Tangent star cones, J. Reine Angew. Math. 483 (1997), 23-59. MR:1431841 (97m:14001) 
23. A. Simis, B. Ulrich and W. V. Vasconcelos, Rees algebras of modules, Proc. London Math. Soc. 87 (2003), 610-646. MR2005877 (2004j:13005)

24. A. Simis and W. V. Vasconcelos, On the dimension and integrality of symmetric algebras, Math. Z. 177 (1981), 341-358. MR618200 (82i:13017)

25. A. Simis and W. V. Vasconcelos, Krull dimension and integrality of symmetric algebras, Manuscripta Math. 61 (1988), 63-78. MR939141 (89e:13011)

26. I. Swanson and C. Huneke, Integral Closure of Ideals, Rings, and Modules, London Math. Soc. Lecture Note Series 336, Cambridge University Press, Cambridge, 2006. MR.2266432 (2008m:13013)

27. W. V. Vasconcelos, Ideals generated by $R$-sequences, J. Algebra 6 (1967), 309-316. MR0213345 (35:4209)

28. W. V. Vasconcelos, Arithmetic of Blowup Algebras, London Math. Soc. Lecture Note Series 195, Cambridge University Press, Cambridge, 1994. MR.1275840 (95g:13005)

29. W. V. Vasconcelos, Integral Closure, Springer Monographs in Mathematics, Springer-Verlag, Berlin, Heidelberg, New York, 2005. MR2153889 (2006m:13007)

30. W. V. Vasconcelos and R. Villarreal, On Gorenstein ideals of codimension four, Proc. Amer. Math. Soc. 98 (1986), 205-210. MR854019 (87k:13041)

31. J. Weyman, Resolutions of the exterior and symmetric powers of a module, J. Algebra $\mathbf{5 8}$ (1979), 333-341. MR540642 (80i:13005)

Departamento de Matemática, Universidade Federal de Pernambuco, 50740-540 ReCIFE, PE, BRAZIL

E-mail address: aron@dmat.ufpe.br

Department of Mathematics, Purdue University, West Lafayette, Indiana 47907-1395

E-mail address: ulrich@math.purdue.edu

Department of Mathematics, Rutgers University, 110 Frelinghuysen Road, PiscatAWAY, NEW JERSEY 08854-8019

E-mail address: vasconce@math.rutgers.edu 